\title{
Sequences of Primitive and Non-primitive BCH Codes
}

\author{
A.S. ANSARI ${ }^{1}$, T. SHAH ${ }^{1}$, ZIA-UR-RAHMAN $^{1}$ and A.A. ANDRADE $^{2}$
}

Received on March 27, 2017 / Accepted on March 21, 2018

\begin{abstract}
In this work, we introduce a method by which it is established that how a sequence of non-primitive $\mathrm{BCH}$ codes can be obtained by a given primitive $\mathrm{BCH}$ code. For this, we rush to the out of routine assembling technique of $\mathrm{BCH}$ codes and use the structure of monoid rings instead of polynomial rings. Accordingly, it is gotten that there is a sequence $\left\{C_{b^{j} n}\right\}_{1 \leq j \leq m}$, where $b^{j} n$ is the length of $C_{b^{j} n}$, of non-primitive binary $\mathrm{BCH}$ codes against a given binary $\mathrm{BCH}$ code $C_{n}$ of length $n$. Matlab based simulated algorithms for encoding and decoding for these type of codes are introduced. Matlab provides in routines for construction of a primitive $\mathrm{BCH}$ code, but impose several constraints, like degree $s$ of primitive irreducible polynomial should be less than 16 . This work focuses on non-primitive irreducible polynomials having degree $b s$, which go far more than 16 .
\end{abstract}

Keywords: Monoid ring, $\mathrm{BCH}$ codes, primitive polynomial, non-primitive polynomial.

\section{INTRODUCTION}

Introducing more general algebraic structures lead to various gains in coding applications and the generality of the algebraic structures helps to find more efficient encoding and decoding algorithms for known codes. This has motivated special attention of many researchers in considering ideals of certain rings [1], [5], [6], [9] and [10]. The extension of a $\mathrm{BCH}$ code embedded in a semigroup ring was considered by Cazaran in [4]. More information regarding ring constructions and its corresponding polynomial codes were given by Kelarev [5]. In [1], the authors elaborated cyclic, BCH, Alternant, Goppa and Srivastava codes over finite rings, which are constructed through a polynomial ring in one indeterminate. Several classes of cyclic codes constructed using the monoid rings are discussed in [2], [14], [13], [12], [11] and [15]. These constructions address the error correction and the code rate in a good way. In [16], Shah et al., showed the existence of a binary cyclic code of length $(n+1) n$ such that a binary $\mathrm{BCH}$ code of length $n$ is embedded in it. Though they were not succeeded to show the existence of binary $\mathrm{BCH}$ code of length

\footnotetext{
*Corresponding author: Antonio A. Andrade - E-mail: antonio.andrade@unesp.br

${ }^{1}$ Department of Mathematics, Quaid-i-Azam University, Islamabad, Pakistan. E-mail: asia_ansari@hotmail.com; stariqshah@gmail.com; ziaagikian@gmail.com

2 Departamento de Matematica, Universidade Estadual Paulista - São José do Rio Preto, SP, Brazil. E-mail: andrade@ibilce.unesp.br
} 
$(n+1) n$ corresponding to a given binary BCH code of length $n$. In [17], a construction method is given by which cyclic codes are ideals in $\mathbb{F}_{2}\left[x ; a \mathbb{Z}_{0}\right]_{n}, \mathbb{F}_{2}[x]_{a n}, \mathbb{F}_{2}\left[x ; \frac{a}{b} \mathbb{Z}_{0}\right]_{b n}$ and $\mathbb{F}_{2}\left[x ; \frac{1}{b} \mathbb{Z}_{0}\right]_{a b n}$, where $\mathbb{Z}_{0}$ is the set of non-negative integers, $a, b \in \mathbb{Z}$ and $a, b>1$. These codes are capable of correcting random as well as burst errors. Moreover, a link between all these codes are also been developed. Furthermore, in [3], the work of [16] is improved and an association between primitive and non-primitive binary BCH codes is obtained by using the monoid ring $\mathbb{F}_{2}\left[x ; \frac{a}{b} \mathbb{Z}_{0}\right]$, where $a, b>1$. It is noticed that the monoid ring $\mathbb{F}_{2}\left[x ; \frac{a}{b} \mathbb{Z}_{0}\right]$ does not contain the polynomial ring $\mathbb{F}_{2}[x]$ for $a>1$. To handle this situation, a BCH code in the monoid ring $\mathbb{F}_{2}\left[x ; a \mathbb{Z}_{0}\right]$ is constructed and then the existence of a non-primitive BCH code in the monoid ring $\mathbb{F}_{2}\left[x ; \frac{a}{b} \mathbb{Z}_{0}\right]$ is showed. The non-primitive $\mathrm{BCH}$ code $C_{b n}$ in the monoid ring $\mathbb{F}_{2}\left[x ; \frac{a}{b} \mathbb{Z}_{0}\right]$ is of length $b n$ and generated by a generalized polynomial $g\left(x^{\frac{a}{b}}\right) \in \mathbb{F}_{2}\left[x ; \frac{a}{b} \mathbb{Z}_{0}\right]$ of degree $b r$. In this line, corresponding to a binary $\mathrm{BCH}$ code $C_{n}$ of length $n$ generated by a generalized polynomial $g\left(x^{a}\right) \in \mathbb{F}_{2}\left[x ; a \mathbb{Z}_{0}\right]$ of degree $r$ it is constructed a code $C_{b n}$ such that $C_{n}$ is embedded in $C_{b n}$.

This work extends the work of [3], where the monoid ring $\mathbb{F}_{2}\left[x ; \frac{a}{b^{j}} \mathbb{Z}_{0}\right]$, with $b=a+i, 1 \leq i, j \leq m$, where $m$ is a positive integer, is used. Corresponding to the sequence $\left\{\mathbb{F}_{2}\left[x ; \frac{a}{b^{j}} \mathbb{Z}_{0}\right]\right\}_{j \geq 1}$ of monoid rings, we obtain a sequence of non-primitive binary $\mathrm{BCH}$ codes $\left\{C_{b^{j}}^{j}\right\}_{j \geq 1}$, based on a primitive BCH code $C_{n}$ of length $n$. The non-primitive BCH code $C_{b^{j} n}^{j}$ in the monoid ring $\mathbb{F}_{2}\left[x ; \frac{a}{b^{j}} \mathbb{Z}_{0}\right]$ is of length $b^{j} n$ and generated by a generalized polynomial $g\left(x^{\frac{a}{b j}}\right) \in \mathbb{F}_{2}\left[x ; \frac{a}{b^{j}} \mathbb{Z}_{0}\right]$ of degree $b^{j} r$. Similarly, corresponding to a given binary $\mathrm{BCH}$ code $C_{n}$ of length $n$ generated by a polynomial $g\left(x^{a}\right) \in \mathbb{F}_{2}\left[x ; a \mathbb{Z}_{0}\right]$ of degree $r$ it is constructed a code $C_{b^{j} n}^{j}$ such that $C_{n}$ is embedded in $C_{b^{j} n}^{j}$, where the length of the binary $\mathrm{BCH}$ code $C_{b^{j} n}^{j}$ is well controlled with better error correction capability. Along with the construction of a sequence $\left\{C_{b^{j} n}^{j}\right\}_{j \geq 1}$ of non-primitive binary BCH codes our focus is on its simulation as well, where the simulation is carried out using Matlab. It provides built in routines only for primitive $\mathrm{BCH}$ codes with degree of primitive polynomial less than 16. Whereas in constructing non-primitive $\mathrm{BCH}$ codes, the degree of non-primitive polynomial goes beyond 16, where to overcome this situation Generic Algorithm is developed in Matlab. The whole method of the algorithm is carried out in two major steps: I) Generating nonprimitive polynomial and II) Error correction in received polynomial. In Table 5, some examples are listed. The non-primitive $\mathrm{BCH}$ codes with same code rate and error corrections are found to be interleaved codes. By interleaving a $t$ random error correcting $(n, k)$ code to degree $\beta$, we obtain a $(\beta n, \beta k)$ code which is capable of correcting any combination of $t$ bursts of length $\beta$ or less [7, Section 9.4], where the non-primitive BCH codes $C_{b^{j} n}^{j}$ have burst error correction capability along with the random error correction.

\section{BCH CODES IN $\mathbb{F}_{2}\left[X ; \frac{A}{B^{J}} \mathbb{Z}_{0}\right]_{B^{J} N}$, WHERE $1 \leq J \leq M$.}

The set of all finitely nonzero functions $f$ from a commutative monoid $(S, *)$ into the binary field $\mathbb{F}_{2}$ is denoted by $\mathbb{F}_{2}(S)$. This set $\mathbb{F}_{2}(S)$ is a ring with respect to binary operations addition and multiplication defined as: $(f+g)(s)=f(s)+g(s)$ and $(f g)(s)=\sum_{t * u=s} f(t) g(u)$, where the symbol $\sum_{t * u=s}$ indicates that the sum is taken over all pairs $(t, u)$ of elements of $S$ such that 
$t * u=s$ and it is understood that in the situation where $s$ is not expressible in the form $t * u$ for any $t, u \in S$, then $(f g)(s)=0$. The ring $\mathbb{F}_{2}(S)$ is known as the monoid ring of $S$ over $\mathbb{F}_{2}$ and it is represented by $\mathbb{F}_{2}[x ; S]$ whenever $S$ is an additive monoid. A nonzero element $f$ of $\mathbb{F}_{2}[x ; S]$ is uniquely represented in the canonical form $\sum_{i=1}^{n} f\left(s_{i}\right) x^{s_{i}}=\sum_{i=1}^{n} f_{i} x^{s_{i}}$, where $f_{i} \neq 0$ and $s_{i} \neq s_{j}$ for $i \neq j$. The monoid ring $\mathbb{F}_{2}[x ; S]$ is a polynomial ring in one indeterminate if $S=\mathbb{Z}_{0}$.

The indeterminate of generalized polynomials in monoid rings $\mathbb{F}_{2}\left[x ; a \mathbb{Z}_{0}\right]$ and $\mathbb{F}_{2}\left[x ; \frac{a}{b^{j}} \mathbb{Z}_{0}\right]$ are given by $x^{a}$ and $x^{\frac{a}{b^{j}}}$ for each $1 \leq j \leq m$, where $m$ is a fix positive integer, and they behave like an indeterminate $x$ in $\mathbb{F}_{2}[x]$. The arbitrary elements in $\mathbb{F}_{2}\left[x ; a \mathbb{Z}_{0}\right]$ and $\mathbb{F}_{2}\left[x ; \frac{a}{b^{j}} \mathbb{Z}_{0}\right]$ are written, respectively, as $f\left(x^{a}\right)=1+\left(x^{a}\right)+\left(x^{a}\right)^{2}+\cdots+\left(x^{a}\right)^{n}$ and $f\left(x^{\frac{a}{b^{j}}}\right)=1+\left(x^{\frac{a}{b^{j}}}\right)+\left(x^{\frac{a}{b^{j}}}\right)^{2}+\cdots\left(x^{\frac{a}{b^{j}}}\right)^{n}$. The monoids $a \mathbb{Z}_{0}$ and $\frac{a}{b^{j}} \mathbb{Z}_{0}$ are totally ordered, so degree and order of elements of $\mathbb{F}_{2}\left[x ; a \mathbb{Z}_{0}\right]$ and $\mathbb{F}_{2}\left[x ; \frac{a}{b^{j}} \mathbb{Z}_{0}\right]$ are defined.

As $\mathbb{F}_{2}\left[x ; a \mathbb{Z}_{0}\right] \subset \mathbb{F}_{2}[x]$, it follows that the construction of BCH code in the factor ring $\mathbb{F}_{2}\left[x ; a \mathbb{Z}_{0}\right]_{n}$ is similar to the construction of a BCH code in $\mathbb{F}_{2}[x]_{n}$. Whereas the construction of BCH codes in $\mathbb{F}_{2}\left[x ; \frac{a}{b^{j}} \mathbb{Z}_{0}\right] /\left(\left(x^{\frac{a}{b^{j}}}\right)^{b^{j} n}-1\right) \equiv \mathbb{F}_{2}\left[x ; \frac{a}{b^{j}} \mathbb{Z}_{0}\right]_{b^{j}{ }_{n}}$ is based on the values of $b$. BCH codes of length $b^{j} n$ in $\mathbb{F}_{2}\left[x ; \frac{a}{b^{j}} \mathbb{Z}_{0}\right]_{b^{j} n}$, corresponding to a BCH code $C_{n}$ of length $n$ don't exist for all values of $b$, where $b=a+i$, such that $1 \leq i \leq m$, and $a, m$ are positive integers. For that, consider the following map $p\left(x^{a}\right)=p_{0}+p_{1} x^{a}+\cdots+p_{n-1}\left(x^{a}\right)^{n-1} \mapsto p_{0}+p_{1}\left(x^{\frac{a}{b^{j}}}\right)^{b^{j}}+\cdots+p_{n-1}\left(x^{\frac{a}{b^{j}}}\right)^{b^{j}(n-1)}=$ $p\left(\begin{array}{c}\frac{a}{b^{j}} \\ \frac{a}{a}\end{array}\right)$, which convert a primitive polynomial $p\left(x^{a}\right)$ of degree $s$ in $\mathbb{F}_{2}\left[x ; a \mathbb{Z}_{0}\right]$ to a polynomial $p\left(x^{\frac{a}{b^{j}}}\right)$ of degree $b^{j} s$ in $\mathbb{F}_{2}\left[x ; \frac{a}{b^{j}} \mathbb{Z}_{0}\right]$. This converted polynomial is found to be non-primitive, but is irreducible for some values of $b$. Therefore, for the construction of a non-primitive BCH code in $\mathbb{F}_{2}\left[x ; \frac{a}{b^{j}} \mathbb{Z}_{0}\right]_{b^{j} n}$, only this specific value of $b$ is selected for which there is an irreducible polynomial $p\left(x^{\frac{a}{b^{j}}}\right)$ in $\mathbb{F}_{2}\left[x ; \frac{a}{b^{j}} \mathbb{Z}_{0}\right]$. Thus, for positive integers $c_{j}, d_{j}$ and $b^{j} n$ such that $2 \leq d_{j} \leq b^{j} n$ with $b^{j} n$ is relatively prime to 2 , there exists a non-primitive binary $\mathrm{BCH}$ code $C_{b^{j} n_{n}}$ of length $b^{j} n$, where $b^{j} n$ is order of an element $\alpha \in \mathbb{F}_{2^{b^{j}}}$.

In Table 1 , we present a list of irreducible polynomials of degree $b^{j} s$ in $\mathbb{F}_{2}\left[x ; \frac{a}{b^{j}} \mathbb{Z}_{0}\right]$ corresponding to primitive irreducible polynomial of degree $s$ in $\mathbb{F}_{2}\left[x ; a \mathbb{Z}_{0}\right]$, where for $p\left(x^{a}\right) \in \mathbb{F}_{2}\left[x ; a \mathbb{Z}_{0}\right]$, $p\left(x^{\frac{a}{b}}\right) \in \mathbb{F}_{2}\left[x ; \frac{a}{b} \mathbb{Z}_{0}\right], p\left(x^{\frac{a}{b^{2}}}\right) \in \mathbb{F}_{2}\left[x ; \frac{a}{b} \mathbb{Z}_{0}\right]$, replace $x^{a}, x^{\frac{a}{b}}$ and $x^{\frac{a}{b^{2}}}$ by $x, y$ and $z$, respectively.

Proposition 1. [3, Proposition 2] If $p\left(x^{a}\right) \in \mathbb{F}_{2}\left[x ; a \mathbb{Z}_{0}\right]$ is a primitive irreducible polynomial of degree $s \in\{2 l, 3 l, 4 l, 6 l\}$, where $l \in \mathbb{Z}_{0}$, then the corresponding generalized polynomial $p\left(x^{\frac{a}{b^{j}}}\right)$ of degree $b^{j}$ s in $\mathbb{F}_{2}\left[x ; \frac{a}{b^{j}} \mathbb{Z}_{0}\right]$ is non-primitive irreducible for $b \in\{3,7,\{3,5\},\{3,7\}\}$, respectively.

Theorem 2. [3, Theorem 3] Let $n=2^{s}-1$ be the length of a primitive BCH code $C_{n}$, where $p\left(x^{a}\right) \in \mathbb{F}_{2}\left[x ; a \mathbb{Z}_{0}\right]$ is a primitive irreducible polynomial of degree s such that $p\left(x^{\frac{a}{b^{j}}}\right) \in \mathbb{F}_{2}\left[x ; \frac{a}{b^{j}} \mathbb{Z}_{0}\right]$ is a non-primitive irreducible polynomial of degree $b^{j}$ s.

1. For positive integers $c_{j}, d_{j}, b^{j} n$ such that $2 \leq d_{j} \leq b^{j} n$ and $b^{j} n$ are relatively prime to 2 , there exist a non-primitive binary $\mathrm{BCH}$ code $C_{b^{j}}$ of length $b^{j} n$, where $b^{j} n$ is the order of an element $\alpha \in \mathbb{F}_{2^{b_{s}}}$. 
Table 1: Irreducible polynomials of degree $b^{j} s$ in $\mathbb{F}_{2}\left[x ; \frac{a}{b^{j}} \mathbb{Z}_{0}\right]$ corresponding to primitive irreducible polynomial of degree $s$ in $\mathbb{F}_{2}\left[x ; a \mathbb{Z}_{0}\right]$.

\begin{tabular}{|c|c|c|}
\hline$p\left(x^{a}\right) \in \mathbb{F}_{2}\left[x ; a \mathbb{Z}_{0}\right]$ & $p\left(x^{\frac{a}{b}}\right) \in \mathbb{F}_{2}\left[x ; \frac{a}{b} \mathbb{Z}_{0}\right]$ & $p\left(x^{\frac{a}{b^{2}}}\right) \in \mathbb{F}_{2}\left[x ; \frac{a}{b} \mathbb{Z}_{0}\right]$ \\
\hline $1+\left(x^{a}\right)+\left(x^{a}\right)^{3}$ & $1+\left(x^{\frac{a}{7}}\right)^{7}+\left(x^{\frac{a}{7}}\right)^{21}$ & $1+\left(x^{\frac{a}{49}}\right)^{49}+\left(x^{\frac{a}{49}}\right)^{147}$ \\
\hline $\begin{array}{l}1+\left(x^{\frac{a}{3}}\right)^{3}+\left(x^{\frac{a}{3}}\right)^{12} \\
1+\left(x^{\frac{a}{5}}\right)^{5}+\left(x^{\frac{a}{5}}\right)^{20}\end{array}$ & $\begin{array}{l}1+\left(x^{\frac{a}{9}}\right)^{9}+\left(x^{\frac{a}{9}}\right)^{36} \\
1+\left(x^{\frac{a}{25}}\right)^{25}+\left(x^{\frac{a}{25}}\right)^{100}\end{array}$ & \\
\hline $1+\left(x^{a}\right)^{3}+\left(x^{a}\right)^{4}$ & $\begin{array}{l}1+\left(x^{\frac{a}{3}}\right)^{9}+\left(x^{\frac{a}{3}}\right)^{12} \\
1+\left(x^{\frac{a}{5}}\right)^{15}+\left(x^{\frac{a}{5}}\right)^{20}\end{array}$ & $\begin{array}{l}1+\left(x^{\frac{a}{9}}\right)^{27}+\left(x^{\frac{a}{9}}\right)^{36} \\
1+\left(x^{\frac{a}{25}}\right)^{75}+\left(x^{\frac{a}{25}}\right)^{100}\end{array}$ \\
\hline $1+\left(x^{a}\right)+\left(x^{a}\right)^{6}$ & $\begin{array}{l}1+\left(x^{\frac{a}{3}}\right)^{3}+\left(x^{\frac{a}{3}}\right)^{18} \\
1+\left(x^{\frac{a}{7}}\right)^{7}+\left(x^{\frac{a}{7}}\right)^{42}\end{array}$ & $\begin{array}{l}1+\left(x^{\frac{a}{9}}\right)^{9}+\left(x^{\frac{a}{9}}\right)^{54} \\
1+\left(x^{\frac{a}{49}}\right)^{49}+\left(x^{\frac{a}{49}}\right)^{294}\end{array}$ \\
\hline $\begin{array}{l}1+\left(x^{a}\right)+\left(x^{a}\right)^{3} \\
+\left(x^{a}\right)^{5}+\left(x^{a}\right)^{8}\end{array}$ & $\begin{array}{l}1+\left(x^{\frac{a}{3}}\right)^{3}+\left(x^{\frac{a}{3}}\right)^{9}+ \\
\left(x^{\frac{a}{3}}\right)^{15}+\left(x^{\frac{a}{3}}\right)^{24} \\
1+\left(x^{\frac{a}{5}}\right)^{5}+\left(x^{\frac{a}{5}}\right)^{15}+ \\
\left(x^{\frac{a}{5}}\right)^{25}+\left(x^{\frac{a}{5}}\right)^{40}\end{array}$ & $\begin{array}{l}1+\left(x^{\frac{a}{9}}\right)^{9}+\left(x^{\frac{a}{9}}\right)^{27}+ \\
\left(x^{\frac{a}{9}}\right)^{45}+\left(x^{\frac{a}{9}}\right)^{72} \\
1+\left(x^{\frac{a}{25}}\right)^{25}+\left(x^{\frac{a}{25}}\right)^{75}+ \\
\left(x^{\frac{a}{25}}\right)^{125}+\left(x^{\frac{a}{25}}\right)^{200}\end{array}$ \\
\hline $1+\left(x^{a}\right)^{4}+\left(x^{a}\right)^{9}$ & $\begin{array}{l}1+\left(x^{\frac{a}{7}}\right)^{28}+\left(x^{\frac{a}{7}}\right)^{63} \\
1+\left(x^{\frac{a}{49}}\right)^{196}+\left(x^{\frac{a}{49}}\right)^{441}\end{array}$ & \\
\hline$\vdots$ & $\vdots$ & $\vdots$ \\
\hline
\end{tabular}

2. The non-primitive $B C H$ code $C_{b^{j} n}$ of length $b^{j} n$ is defined as

$$
C_{b^{j_{n}}}=\left\{v\left(x^{\frac{a}{b^{j}}}\right) \in \mathbb{F}_{2}\left[x ; \frac{a}{b^{j}} \mathbb{Z}_{0}\right]_{b^{j_{n}}}: v\left(\alpha^{i}\right)=0 \text { for all } i=c_{j}, c_{j}+1, \cdots, c_{j}+d_{j}-2 .\right.
$$

Equivalently, $C_{b^{j_{n}}}$ is the null space of the matrix

$$
H=\left[\begin{array}{ccccc}
1 & \alpha^{c_{j}} & \alpha^{2 c_{j}} & \cdots & \alpha^{(b n-1) c_{j}} \\
1 & \alpha^{c_{j}+1} & \alpha^{2\left(c_{j}+1\right)} & \cdots & \alpha^{(b n-1)\left(c_{j}+1\right)} \\
\vdots & \vdots & \vdots & \ddots & \vdots \\
1 & \alpha^{c_{j}+d_{j}-2} & \alpha^{2\left(c_{j}+d_{j}-2\right)} & \cdots & \alpha^{(b n-1)\left(c_{j}+d_{j}-2\right)}
\end{array}\right]
$$

The following example illustrates the construction of a non-primitive $\mathrm{BCH}$ code of length $3^{2} n$ through $\mathbb{F}_{2}\left[x ; \frac{2}{3^{2}} \mathbb{Z}_{0}\right]$.

Example 2.1. Corresponding to a primitive polynomial $p\left(x^{2}\right)=1+\left(x^{2}\right)+\left(x^{2}\right)^{4}$ in $\mathbb{F}_{2}\left[x ; 2 \mathbb{Z}_{0}\right]$ there is a non-primitive irreducible polynomial $p\left(x^{\frac{2}{3^{2}}}\right)=1+\left(x^{\frac{2}{9}}\right)^{9}+\left(x^{\frac{2}{9}}\right)^{36}$ in $\mathbb{F}_{2}\left[x ; \frac{2}{3^{2}} \mathbb{Z}_{0}\right]$ (Table 
1). Let $\alpha \in G F\left(2^{36}\right)$ such that $\alpha$ satisfies the relation $\alpha^{36}+\alpha^{9}+1=0$. Using this relation, in the Table 2, we obtain the distinct elements of $G F\left(2^{36}\right)$.

By Table 2, it follows that $b^{2} n=3^{2} \times 15=135$. Now, to calculate the generating polynomial $g\left(x^{\frac{2}{9}}\right)$, first calculate the minimal polynomials. By [8, Theorem 4.4.2], the following roots

$$
\begin{aligned}
& \alpha, \alpha^{2}, \alpha^{4}, \alpha^{8}, \alpha^{16}, \alpha^{32}, \alpha^{64}, \alpha^{128}, \alpha^{121}, \alpha^{107}, \alpha^{79}, \alpha^{23}, \alpha^{46}, \alpha^{92}, \alpha^{49}, \alpha^{98}, \alpha^{61}, \alpha^{122}, \\
& \alpha^{109}, \alpha^{83}, \alpha^{31}, \alpha^{62}, \alpha^{124}, \alpha^{113}, \alpha^{91}, \alpha^{47}, \alpha^{94}, \alpha^{53}, \alpha^{106}, \alpha^{77}, \alpha^{19} \alpha^{38}, \alpha^{76}, \alpha^{17}, \alpha^{34}, \alpha^{68}
\end{aligned}
$$

have same minimal polynomial $m_{1}\left(x^{\frac{2}{9}}\right)=p\left(x^{\frac{2}{9}}\right)=1+\left(x^{\frac{2}{9}}\right)^{9}+\left(x^{\frac{2}{9}}\right)^{36}$. It $m_{3}\left(x^{\frac{2}{9}}\right)$ is the minimal polynomial for $\alpha^{3}$, then $\alpha^{3}, \alpha^{6}, \alpha^{12}, \alpha^{24}, \alpha^{48}, \alpha^{96}, \alpha^{57}, \alpha^{114}, \alpha^{93}, \alpha^{51}, \alpha^{102}$ and $\alpha^{69}$ all are roots for $m_{3}\left(x^{\frac{2}{9}}\right)$. Therefore, it follows that $m_{3}\left(x^{\frac{2}{9}}\right)=\left(x^{\frac{2}{9}}\right)^{12}+\left(x^{\frac{2}{9}}\right)^{3}+1$. Similarly,

$$
\begin{aligned}
& m_{5}\left(x^{\frac{2}{9}}\right)=\left(x^{\frac{2}{9}}\right)^{18}+\left(x^{\frac{2}{9}}\right)^{9}+1, m_{7}\left(x^{\frac{2}{9}}\right)=\left(x^{\frac{2}{9}}\right)^{36}+\left(x^{\frac{2}{9}}\right)^{27}+1 \\
& m_{9}\left(x^{\frac{2}{9}}\right)=\left(x^{\frac{2}{9}}\right)^{4}+\left(x^{\frac{2}{9}}\right)+1, m_{15}\left(x^{\frac{2}{9}}\right)=\left(x^{\frac{2}{9}}\right)^{6}+\left(x^{\frac{2}{9}}\right)^{3}+1 \\
& m_{21}\left(x^{\frac{2}{9}}\right)=\left(x^{\frac{2}{9}}\right)^{12}+\left(x^{\frac{2}{9}}\right)^{9}+1 \\
& m_{27}\left(x^{\frac{2}{9}}\right)=\left(x^{\frac{2}{9}}\right)^{4}+\left(x^{\frac{2}{9}}\right)^{3}+\left(x^{\frac{2}{9}}\right)^{2}+\left(x^{\frac{2}{9}}\right)+1 \\
& m_{45}\left(x^{\frac{2}{9}}\right)=\left(x^{\frac{2}{9}}\right)^{2}+\left(x^{\frac{2}{9}}\right)+1, m_{63}\left(x^{\frac{2}{9}}\right)=\left(x^{\frac{2}{9}}\right)^{4}+\left(x^{\frac{2}{9}}\right)^{3}+1 .
\end{aligned}
$$

The BCH code with $d_{2}=3$ has generator polynomial $g\left(x^{\frac{2}{9}}\right)=1+\left(x^{\frac{2}{9}}\right)^{9}+\left(x^{\frac{2}{9}}\right)^{36}$. It corrects up to 1 error and its code rate is $\frac{99}{135}=0.733$. The $B C H$ code with $d_{2}=5$ has generator polynomial

$$
\begin{aligned}
g\left(x^{\frac{2}{9}}\right) & =\left(m_{1}\left(x^{\frac{2}{9}}\right)\right)\left(m_{3}\left(x^{\frac{2}{9}}\right)\right)=\left(\left(x^{\frac{2}{9}}\right)^{36}+\left(x^{\frac{2}{9}}\right)^{9}+1\right)\left(\left(x^{\frac{2}{9}}\right)^{12}+\left(x^{\frac{2}{9}}\right)^{3}+1\right) \\
& =\left(x^{\frac{2}{9}}\right)^{48}+\left(x^{\frac{2}{9}}\right)^{39}+\left(x^{\frac{2}{9}}\right)^{36}+\left(x^{\frac{2}{9}}\right)^{21}+\left(x^{\frac{2}{9}}\right)^{9}+\left(x^{\frac{2}{9}}\right)^{3}+1 .
\end{aligned}
$$

It corrects up to 3 errors and its code rate is $\frac{87}{135}=0.644$. Similarly, the BCH codes with $d_{2}=$ $7,9,15,21,27,45,63$ and 135 have generator polynomials

$$
\begin{aligned}
g\left(x^{\frac{2}{9}}\right)= & \left(m_{1}\left(x^{\frac{2}{9}}\right)\right)\left(m_{3}\left(x^{\frac{2}{9}}\right)\right)\left(m_{5}\left(x^{\frac{2}{9}}\right)\right) \\
= & \left(x^{\frac{2}{9}}\right)^{66}+\left(x^{\frac{2}{9}}\right)^{54}+\left(x^{\frac{2}{9}}\right)^{45}+\left(x^{\frac{2}{9}}\right)^{36}+\left(x^{\frac{2}{9}}\right)^{30}+\left(x^{\frac{2}{9}}\right)^{27}+\left(x^{\frac{2}{9}}\right)^{12}+\left(x^{\frac{2}{9}}\right)^{3}+1 . \\
g\left(x^{\frac{2}{9}}\right)= & \left(m_{1}\left(x^{\frac{2}{9}}\right)\right)\left(m_{3}\left(x^{\frac{2}{9}}\right)\right)\left(m_{5}\left(x^{\frac{2}{9}}\right)\right)\left(m_{7}\left(x^{\frac{2}{9}}\right)\right) \\
= & \left(x^{\frac{2}{9}}\right)^{102}+\left(x^{\frac{2}{9}}\right)^{93}+\left(x^{\frac{2}{9}}\right)^{90}+\left(x^{\frac{2}{9}}\right)^{57}+\left(x^{\frac{2}{9}}\right)^{48}+\left(x^{\frac{2}{9}}\right)^{45}+\left(x^{\frac{2}{9}}\right)^{12}+\left(x^{\frac{2}{9}}\right)^{3}+1 . \\
g\left(x^{\frac{2}{9}}\right)= & \left(m_{1}\left(x^{\frac{2}{9}}\right)\right)\left(m_{3}\left(x^{\frac{2}{9}}\right)\right)\left(m_{5}\left(x^{\frac{2}{9}}\right)\right)\left(m_{7}\left(x^{\frac{2}{9}}\right)\right)\left(m_{9}\left(x^{\frac{2}{9}}\right)\right) \\
= & \left(x^{\frac{2}{9}}\right)^{106}+\left(x^{\frac{2}{9}}\right)^{103}+\left(x^{\frac{2}{9}}\right)^{102}+\left(x^{\frac{2}{9}}\right)^{97}+\left(x^{\frac{2}{9}}\right)^{93}+\left(x^{\frac{2}{9}}\right)^{91}+\left(x^{\frac{2}{9}}\right)^{90}+\left(x^{\frac{2}{9}}\right)^{61} \\
& +\left(x^{\frac{2}{9}}\right)^{58}+\left(x^{\frac{2}{9}}\right)^{57}+\left(x^{\frac{2}{9}}\right)^{52}+\left(x^{\frac{2}{9}}\right)^{48}+\left(x^{\frac{2}{9}}\right)^{46}+\left(x^{\frac{2}{9}}\right)^{45}+\left(x^{\frac{2}{9}}\right)^{16}+\left(x^{\frac{2}{9}}\right)^{13} \\
& +\left(x^{\frac{2}{9}}\right)^{12}+\left(x^{\frac{2}{9}}\right)^{7}+\left(x^{\frac{2}{9}}\right)^{3}+\left(x^{\frac{2}{9}}\right)+1 . \\
g\left(x^{\frac{2}{9}}\right)= & \left(m_{1}\left(x^{\frac{2}{9}}\right)\right)\left(m_{3}\left(x^{\frac{2}{9}}\right)\right)\left(m_{5}\left(x^{\frac{2}{9}}\right)\right)\left(m_{7}\left(x^{\frac{2}{9}}\right)\right)\left(m_{9}\left(x^{\frac{2}{9}}\right)\right)\left(m_{15}\left(x^{9}\right)\right) \\
& \left(x^{\frac{2}{9}}\right)^{112}+\left(x^{\frac{2}{9}}\right)^{108}+\left(x^{\frac{2}{9}}\right)^{105}+\left(x^{\frac{2}{9}}\right)^{102}+\left(x^{\frac{2}{9}}\right)^{100}+\left(x^{\frac{2}{9}}\right)^{99}+\left(x^{\frac{2}{9}}\right)^{94}+\left(x^{\frac{2}{9}}\right)^{91} \\
& +\left(x^{\frac{2}{9}}\right)^{90}+\left(x^{\frac{2}{9}}\right)^{67}+\left(x^{\frac{2}{9}}\right)^{63}+\left(x^{\frac{2}{9}}\right)^{60}+\left(x^{\frac{2}{9}}\right)^{57}+\left(x^{\frac{2}{9}}\right)^{55}+\left(x^{\frac{2}{9}}\right)^{54}+\left(x^{\frac{2}{9}}\right)^{49} \\
& +\left(x^{\frac{2}{9}}\right)^{46}+\left(x^{\frac{2}{9}}\right)^{45}+\left(x^{\frac{2}{9}}\right)^{22}+\left(x^{\frac{2}{9}}\right)^{18}+\left(x^{\frac{2}{9}}\right)^{15}+\left(x^{\frac{2}{9}}\right)^{12}+\left(x^{\frac{2}{9}}\right)^{10}+\left(x^{\frac{2}{9}}\right)^{9} \\
& +\left(x^{\frac{2}{9}}\right)^{4}+\left(x^{\frac{2}{9}}\right)+1 . \\
&
\end{aligned}
$$


Table 2: Distinct elements of $G F\left(2^{36}\right)$.

\begin{tabular}{|c|c|c|c|}
\hline$\alpha^{36}=1+\alpha^{9}$ & $\alpha^{62}=\alpha^{26}+\alpha^{35}$ & $\alpha^{88}=\alpha^{16}+\alpha^{34}$ & $\alpha^{114}=\alpha^{6}+\alpha^{15}+\alpha^{24}+\alpha^{33}$ \\
\hline$\alpha^{37}=\alpha+\alpha^{10}$ & $\alpha^{63}=1+\alpha^{9}+\alpha^{27}$ & $\alpha^{89}=\alpha^{17}+\alpha^{35}$ & $\alpha^{115}=\alpha^{7}+\alpha^{16}+\alpha^{25}+\alpha^{34}$ \\
\hline$\alpha^{38}=\alpha^{2}+\alpha^{11}$ & $\alpha^{64}=\alpha+\alpha^{10}+\alpha^{28}$ & $\alpha^{90}=1+\alpha^{9}+\alpha^{18}$ & $\alpha^{116}=\alpha^{8}+\alpha^{17}+\alpha^{26}+\alpha^{35}$ \\
\hline$\alpha^{39}=\alpha^{3}+\alpha^{12}$ & $\alpha^{65}=\alpha^{2}+\alpha^{11}+\alpha^{29}$ & $\alpha^{91}=\alpha+\alpha^{10}+\alpha^{19}$ & $\alpha^{117}=1+\alpha^{18}+\alpha^{27}$ \\
\hline$\alpha^{40}=\alpha^{4}+\alpha^{13}$ & $\alpha^{66}=\alpha^{3}+\alpha^{12}+\alpha^{30}$ & $\alpha^{92}=\alpha^{2}+\alpha^{11}+\alpha^{20}$ & $\alpha^{118}=\alpha+\alpha^{19}+\alpha^{28}$ \\
\hline$\alpha^{41}=\alpha^{5}+\alpha^{14}$ & $\alpha^{67}=\alpha^{4}+\alpha^{13}+\alpha^{31}$ & $\alpha^{93}=\alpha^{3}+\alpha^{12}+\alpha^{21}$ & $\alpha^{119}=\alpha^{2}+\alpha^{20}+\alpha^{29}$ \\
\hline$\alpha^{42}=\alpha^{6}+\alpha^{15}$ & $\alpha^{68}=\alpha^{5}+\alpha^{14}+\alpha^{32}$ & $\alpha^{94}=\alpha^{4}+\alpha^{13}+\alpha^{22}$ & $\alpha^{120}=\alpha^{3}+\alpha^{21}+\alpha^{30}$ \\
\hline$\alpha^{43}=\alpha^{7}+\alpha^{16}$ & $\alpha^{69}=\alpha^{6}+\alpha^{15}+\alpha^{33}$ & $\alpha^{95}=\alpha^{5}+\alpha^{14}+\alpha^{23}$ & $\alpha^{121}=\alpha^{4}+\alpha^{22}+\alpha^{31}$ \\
\hline$\alpha^{44}=\alpha^{8}+\alpha^{17}$ & $\alpha^{70}=\alpha^{7}+\alpha^{16}+\alpha^{34}$ & $\alpha^{96}=\alpha^{6}+\alpha^{15}+\alpha^{24}$ & $\alpha^{122}=\alpha^{5}+\alpha^{23}+\alpha^{32}$ \\
\hline$\alpha^{45}=\alpha^{9}+\alpha^{18}$ & $\alpha^{71}=\alpha^{8}+\alpha^{17}+\alpha^{35}$ & $\alpha^{97}=\alpha^{7}+\alpha^{16}+\alpha^{25}$ & $\alpha^{123}=\alpha^{6}+\alpha^{24}+\alpha^{33}$ \\
\hline$\alpha^{46}=\alpha^{10}+\alpha^{19}$ & $\alpha^{72}=1+\alpha^{18}$ & $\alpha^{98}=\alpha^{8}+\alpha^{17}+\alpha^{26}$ & $\alpha^{124}=\alpha^{7}+\alpha^{25}+\alpha^{34}$ \\
\hline$\alpha^{47}=\alpha^{11}+\alpha^{20}$ & $\alpha^{73}=\alpha+\alpha^{19}$ & $\alpha^{99}=\alpha^{9}+\alpha^{18}+\alpha^{27}$ & $\alpha^{125}=\alpha^{8}+\alpha^{26}+\alpha^{35}$ \\
\hline$\alpha^{48}=\alpha^{12}+\alpha^{21}$ & $\alpha^{74}=\alpha^{2}+\alpha^{20}$ & $\alpha^{100}=\alpha^{10}+\alpha^{19}+\alpha^{28}$ & $\alpha^{126}=1+\alpha^{27}$ \\
\hline$\alpha^{49}=\alpha^{13}+\alpha^{22}$ & $\alpha^{75}=\alpha^{3}+\alpha^{21}$ & $\alpha^{101}=\alpha^{11}+\alpha^{20}+\alpha^{29}$ & $\alpha^{127}=\alpha+\alpha^{28}$ \\
\hline$\alpha^{50}=\alpha^{14}+\alpha^{23}$ & $\alpha^{76}=\alpha^{4}+\alpha^{22}$ & $\alpha^{102}=\alpha^{12}+\alpha^{21}+\alpha^{30}$ & $\alpha^{128}=\alpha^{2}+\alpha^{29}$ \\
\hline$\alpha^{51}=\alpha^{15}+\alpha^{24}$ & $\alpha^{77}=\alpha^{5}+\alpha^{23}$ & $\alpha^{103}=\alpha^{13}+\alpha^{22}+\alpha^{31}$ & $\alpha^{129}=\alpha^{3}+\alpha^{30}$ \\
\hline$\alpha^{52}=\alpha^{16}+\alpha^{25}$ & $\alpha^{78}=\alpha^{6}+\alpha^{24}$ & $\alpha^{104}=\alpha^{14}+\alpha^{23}+\alpha^{32}$ & $\alpha^{130}=\alpha^{4}+\alpha^{31}$ \\
\hline$\alpha^{53}=\alpha^{17}+\alpha^{26}$ & $\alpha^{79}=\alpha^{7}+\alpha^{25}$ & $\alpha^{105}=\alpha^{15}+\alpha^{24}+\alpha^{33}$ & $\alpha^{131}=\alpha^{5}+\alpha^{32}$ \\
\hline$\alpha^{54}=\alpha^{18}+\alpha^{27}$ & $\alpha^{80}=\alpha^{8}+\alpha^{26}$ & $\alpha^{106}=\alpha^{16}+\alpha^{25}+\alpha^{34}$ & $\alpha^{132}=\alpha^{6}+\alpha^{33}$ \\
\hline$\alpha^{55}=\alpha^{19}+\alpha^{28}$ & $\alpha^{81}=\alpha^{9}+\alpha^{27}$ & $\alpha^{107}=\alpha^{17}+\alpha^{26}+\alpha^{35}$ & $\alpha^{133}=\alpha^{7}+\alpha^{34}$ \\
\hline$\alpha^{56}=\alpha^{20}+\alpha^{29}$ & $\alpha^{82}=\alpha^{10}+\alpha^{28}$ & $\alpha^{108}=1+\alpha^{9}+\alpha^{18}+\alpha^{27}$ & $\alpha^{134}=\alpha^{8}+\alpha^{35}$ \\
\hline$\alpha^{57}=\alpha^{21}+\alpha^{30}$ & $\alpha^{83}=\alpha^{11}+\alpha^{29}$ & $\alpha^{109}=\alpha+\alpha^{10}+\alpha^{19}+\alpha^{28}$ & $\alpha^{135}=1$ \\
\hline$\alpha^{58}=\alpha^{22}+\alpha^{31}$ & $\alpha^{84}=\alpha^{12}+\alpha^{30}$ & $\alpha^{110}=\alpha^{2}+\alpha^{11}+\alpha^{20}+\alpha^{29}$ & \\
\hline$\alpha^{59}=\alpha^{23}+\alpha^{32}$ & $\alpha^{85}=\alpha^{13}+\alpha^{31}$ & $\alpha^{111}=\alpha^{3}+\alpha^{12}+\alpha^{21}+\alpha^{30}$ & \\
\hline$\alpha^{60}=\alpha^{24}+\alpha^{33}$ & $\alpha^{86}=\alpha^{14}+\alpha^{32}$ & $\alpha^{112}=\alpha^{4}+\alpha^{13}+\alpha^{22}+\alpha^{31}$ & \\
\hline$\alpha^{61}=\alpha^{25}+\alpha^{34}$ & $\alpha^{87}=\alpha^{15}+\alpha^{33}$ & $\alpha^{113}=\alpha^{5}+\alpha^{14}+\alpha^{23}+\alpha^{32}$ & \\
\hline
\end{tabular}




$$
\begin{aligned}
g\left(x^{\frac{2}{9}}\right)= & \left(m_{1}\left(x^{\frac{2}{9}}\right)\right)\left(m_{3}\left(x^{\frac{2}{9}}\right)\right)\left(m_{5}\left(x^{\frac{2}{9}}\right)\right)\left(m_{7}\left(x^{\frac{2}{9}}\right)\right)\left(m_{9}\left(x^{\frac{2}{9}}\right)\right)\left(m_{15}\left(x^{\frac{2}{9}}\right)\right)\left(m_{21}\left(x^{\frac{2}{9}}\right)\right) \\
= & \left(x^{\frac{2}{9}}\right)^{124}+\left(x^{\frac{2}{9}}\right)^{121}+\left(x^{\frac{2}{9}}\right)^{120}+\left(x^{\frac{2}{9}}\right)^{109}+\left(x^{\frac{2}{9}}\right)^{106}+\left(x^{\frac{2}{9}}\right)^{105}+\left(x^{\frac{2}{9}}\right)^{94}+\left(x^{\frac{2}{9}}\right)^{91} \\
& +\left(x^{\frac{2}{9}}\right)^{90}+\left(x^{\frac{2}{9}}\right)^{79}+\left(x^{\frac{2}{9}}\right)^{76}+\left(x^{\frac{2}{9}}\right)^{75}+\left(x^{\frac{2}{9}}\right)^{64}+\left(x^{\frac{2}{9}}\right)^{61}+\left(x^{\frac{2}{9}}\right)^{60}+\left(x^{\frac{2}{9}}\right)^{49} \\
& +\left(x^{\frac{2}{9}}\right)^{46}+\left(x^{\frac{2}{9}}\right)^{45}+\left(x^{\frac{2}{9}}\right)^{34}+\left(x^{\frac{2}{9}}\right)^{31}+\left(x^{\frac{2}{9}}\right)^{30}+\left(x^{\frac{2}{9}}\right)^{19}+\left(x^{\frac{2}{9}}\right)^{16}+\left(x^{\frac{2}{9}}\right)^{15} \\
& +\left(x^{\frac{2}{9}}\right)^{4}+\left(x^{\frac{2}{9}}\right)+1 .
\end{aligned}
$$

$$
\begin{aligned}
g\left(x^{\frac{2}{9}}\right)= & \left(m_{1}\left(x^{\frac{2}{9}}\right)\right)\left(m_{3}\left(x^{\frac{2}{9}}\right)\right)\left(m_{5}\left(x^{\frac{2}{9}}\right)\right)\left(m_{7}\left(x^{\frac{2}{9}}\right)\right)\left(m_{9}\left(x^{\frac{2}{9}}\right)\right)\left(m_{15}\left(x^{\frac{2}{9}}\right)\right)\left(m_{21}\left(x^{\frac{2}{9}}\right)\right)\left(m_{27}\left(x^{\frac{2}{9}}\right)\right) \\
= & \left(x^{\frac{2}{9}}\right)^{128}+\left(x^{\frac{2}{9}}\right)^{127}+\left(x^{\frac{2}{9}}\right)^{126}+\left(x^{\frac{2}{9}}\right)^{124}+\left(x^{\frac{2}{9}}\right)^{120}+\left(x^{\frac{2}{9}}\right)^{113}+\left(x^{\frac{2}{9}}\right)^{112}+\left(x^{\frac{2}{9}}\right)^{111} \\
& +\left(x^{\frac{2}{9}}\right)^{109}+\left(x^{\frac{2}{9}}\right)^{105}+\left(x^{\frac{2}{9}}\right)^{98}+\left(x^{\frac{2}{9}}\right)^{97}+\left(x^{\frac{2}{9}}\right)^{96}+\left(x^{\frac{2}{9}}\right)^{94}+\left(x^{\frac{2}{9}}\right)^{90}+\left(x^{\frac{2}{9}}\right)^{83}+\left(x^{\frac{2}{9}}\right)^{82} \\
& +\left(x^{\frac{2}{9}}\right)^{81}+\left(x^{\frac{2}{9}}\right)^{79}+\left(x^{\frac{2}{9}}\right)^{75}+\left(x^{\frac{2}{9}}\right)^{68}+\left(x^{\frac{2}{9}}\right)^{67}+\left(x^{\frac{2}{9}}\right)^{66}+\left(x^{\frac{2}{9}}\right)^{64}+\left(x^{\frac{2}{9}}\right)^{60}+\left(x^{\frac{2}{9}}\right)^{53} \\
& +\left(x^{\frac{2}{9}}\right)^{52}+\left(x^{\frac{2}{9}}\right)^{51}+\left(x^{\frac{2}{9}}\right)^{49}+\left(x^{\frac{2}{9}}\right)^{45}+\left(x^{\frac{2}{9}}\right)^{38}+\left(x^{\frac{2}{9}}\right)^{37}+\left(x^{\frac{2}{9}}\right)^{36}+\left(x^{\frac{2}{9}}\right)^{34}+\left(x^{\frac{2}{9}}\right)^{30} \\
& +\left(x^{\frac{2}{9}}\right)^{23}+\left(x^{\frac{2}{9}}\right)^{22}+\left(x^{\frac{2}{9}}\right)^{21}+\left(x^{\frac{2}{9}}\right)^{19}+\left(x^{\frac{2}{9}}\right)^{15}+\left(x^{\frac{2}{9}}\right)^{8}+\left(x^{\frac{2}{9}}\right)^{7}+\left(x^{\frac{2}{9}}\right)^{6}+\left(x^{\frac{2}{9}}\right)^{4}+1 .
\end{aligned}
$$

$$
\begin{aligned}
g\left(x^{\frac{2}{9}}\right)= & \left(m_{1}\left(x^{\frac{2}{9}}\right)\right)\left(m_{3}\left(x^{\frac{2}{9}}\right)\right)\left(m_{5}\left(x^{\frac{2}{9}}\right)\right)\left(m_{7}\left(x^{\frac{2}{9}}\right)\right)\left(m_{9}\left(x^{\frac{2}{9}}\right)\right)\left(m_{15}\left(x^{\frac{2}{9}}\right)\right)\left(m _ { 2 1 } \left(x^{\frac{2}{9}}\right.\right. \\
& )\left(m_{27}\left(x^{\frac{2}{9}}\right)\right)\left(m_{45}\left(x^{\frac{2}{9}}\right)\right)=\left(x^{\frac{2}{9}}\right)^{130}+\left(x^{\frac{2}{9}}\right)^{128}+\left(x^{\frac{2}{9}}\right)^{125}+\left(x^{\frac{2}{9}}\right)^{124}+\left(x^{\frac{2}{9}}\right)^{122} \\
& +\left(x^{\frac{2}{9}}\right)^{121}+\left(x^{\frac{2}{9}}\right)^{120}+\left(x^{\frac{2}{9}}\right)^{115}+\left(x^{\frac{2}{9}}\right)^{113}+\left(x^{\frac{2}{9}}\right)^{110}+\left(x^{\frac{2}{9}}\right)^{109}+\left(x^{\frac{2}{9}}\right)^{107} \\
& +\left(x^{\frac{2}{9}}\right)^{106}+\left(x^{\frac{2}{9}}\right)^{105}+\left(x^{\frac{2}{9}}\right)^{100}+\left(x^{\frac{2}{9}}\right)^{98}+\left(x^{\frac{2}{9}}\right)^{95}+\left(x^{\frac{2}{9}}\right)^{94}+\left(x^{\frac{2}{9}}\right)^{92} \\
& +\left(x^{\frac{2}{9}}\right)^{91}+\left(x^{\frac{2}{9}}\right)^{90}+\left(x^{\frac{2}{9}}\right)^{85}+\left(x^{\frac{2}{9}}\right)^{83}+\left(x^{\frac{2}{9}}\right)^{80}+\left(x^{\frac{2}{9}}\right)^{79}+\left(x^{\frac{2}{9}}\right)^{77}+\left(x^{\frac{2}{9}}\right)^{76} \\
& +\left(x^{\frac{2}{9}}\right)^{75}+\left(x^{\frac{2}{9}}\right)^{70}+\left(x^{\frac{2}{9}}\right)^{68}+\left(x^{\frac{2}{9}}\right)^{65}+\left(x^{\frac{2}{9}}\right)^{64}+\left(x^{\frac{2}{9}}\right)^{62}+\left(x^{\frac{2}{9}}\right)^{61}+\left(x^{\frac{2}{9}}\right)^{60} \\
& +\left(x^{\frac{2}{9}}\right)^{55}+\left(x^{\frac{2}{9}}\right)^{53}+\left(x^{\frac{2}{9}}\right)^{50}+\left(x^{\frac{2}{9}}\right)^{49}+\left(x^{\frac{2}{9}}\right)^{47}+\left(x^{\frac{2}{9}}\right)^{46}+\left(x^{\frac{2}{9}}\right)^{45}+\left(x^{\frac{2}{9}}\right)^{40} \\
& +\left(x^{\frac{2}{9}}\right)^{38}+\left(x^{\frac{2}{9}}\right)^{35}+\left(x^{\frac{2}{9}}\right)^{34}+\left(x^{\frac{2}{9}}\right)^{32}+\left(x^{\frac{2}{9}}\right)^{31}+\left(x^{\frac{2}{9}}\right)^{30}+\left(x^{\frac{2}{9}}\right)^{25} \\
& +\left(x^{\frac{2}{9}}\right)^{23}+\left(x^{\frac{2}{9}}\right)^{20}+\left(x^{\frac{2}{9}}\right)^{19}+\left(x^{\frac{2}{9}}\right)^{17}+\left(x^{\frac{2}{9}}\right)^{16}+\left(x^{\frac{2}{9}}\right)^{15}+\left(x^{\frac{2}{9}}\right)^{10}+\left(x^{\frac{2}{9}}\right)^{8} \\
& +\left(x^{\frac{2}{9}}\right)^{5}+\left(x^{\frac{2}{9}}\right)^{4}+\left(x^{\frac{2}{9}}\right)^{2}+\left(x^{\frac{2}{9}}\right)+1 .
\end{aligned}
$$

$$
\begin{aligned}
g\left(x^{\frac{2}{9}}\right)= & \left.m_{1}\left(x^{\frac{2}{9}}\right)\right)\left(m_{3}\left(x^{\frac{2}{9}}\right)\right)\left(m_{5}\left(x^{\frac{2}{9}}\right)\right)\left(m_{7}\left(x^{\frac{2}{9}}\right)\right)\left(m_{9}\left(x^{\frac{2}{9}}\right)\right)\left(m_{15}\left(x^{\frac{2}{9}}\right)\right) \\
& \left(m_{21}\left(x^{\frac{2}{9}}\right)\right)\left(m_{27}\left(x^{\frac{2}{9}}\right)\right)\left(m_{45}\left(x^{\frac{2}{9}}\right)\right)\left(m_{63}\left(x^{\frac{2}{9}}\right)\right) \\
= & \left(x^{\frac{2}{9}}\right)^{134}+\left(x^{\frac{2}{9}}\right)^{133}+\cdots+\left(x^{\frac{2}{9}}\right)^{2}+\left(x^{\frac{2}{9}}\right)+1 .
\end{aligned}
$$

Finally, errors like 3,4,7,10,13,22,31 and 67 are corrected.

From Example 2.1 and [3, Example 1], it follows that the code generated through $\mathbb{F}_{2}\left[x ; \frac{a}{b^{j}} \mathbb{Z}_{0}\right]$ corrects more errors and has better code rate than the code generated through $\mathbb{F}_{2}\left[x ; a \mathbb{Z}_{0}\right]$. Now, we are in position to develop a link between a primitive $(n, n-r)$ binary $\mathrm{BCH}$ code $C_{n}$ and a nonprimitive $\left(b^{j} n, b^{j} n-r_{j}\right)$ binary BCH code $C_{b_{j} j_{n}}$, where $r$ and $r_{j}$ are, respectively, the degrees of their generating polynomials $g\left(x^{a}\right)$ and $g\left(x^{\frac{a}{b^{j}}}\right)$. From Theorem 2, it follows that the generalized polynomial $g\left(x^{\frac{a}{b^{j}}}\right)$ in $\mathbb{F}_{2}\left[x ; \frac{a}{b^{j}} \mathbb{Z}_{0}\right]$ divides $\left(x^{\frac{a}{b^{j}}}\right)^{b^{j} n}-1$ in $\mathbb{F}_{2}\left[x ; \frac{a}{b^{j}} \mathbb{Z}_{0}\right]$. So, there is a non-primitive BCH code $C_{b^{j} n}$ generated by $g\left(x^{\frac{a}{b^{j}}}\right)$ in $\mathbb{F}_{2}\left[x ; \frac{a}{b^{j}} \mathbb{Z}_{0}\right]_{b^{j} n_{n}}$. By the same argument, as $b^{j} n$ divides 
$n_{j}=2^{b^{j} s}-1$, it follows that $\left(x^{\frac{a}{b^{j}}}\right)^{b^{j} n}-1$ divides $\left(x^{\frac{a}{b^{j}}}\right)^{n_{j}}-1$ in $\mathbb{F}_{2}\left[x ; \frac{a}{b^{j}} \mathbb{Z}_{0}\right]$. Therefore, $\left(\left(x^{\frac{a}{b^{j}}}\right)^{n_{j}}-\right.$ 1) $\subset\left(\left(x^{\frac{a}{b^{j}}}\right)^{b^{j} n}-1\right)$. Consequently, from third isomorphism theorem for rings, it follows that

$$
\frac{\mathbb{F}_{2}\left[x ; \frac{a}{b^{j}} \mathbb{Z}_{0}\right] /\left(\left(x^{\frac{a}{b^{j}}}\right)^{n_{j}}-1\right)}{\left(\left(x^{\frac{a}{b^{j}}}\right)^{b^{j_{n}}}-1\right) /\left(\left(x^{\frac{a}{b^{j}}}\right)^{n_{j}}-1\right)} \simeq \frac{\mathbb{F}_{2}\left[x ; \frac{a}{b^{j}} \mathbb{Z}_{0}\right]}{\left(\left(x^{\frac{a}{b^{j}}}\right)^{b^{j}}-1\right)} \simeq \frac{\mathbb{F}_{2}\left[x ; a \mathbb{Z}_{0}\right]}{\left(\left(x^{a}\right)^{n}-1\right)}
$$

Thus, there are embeddings $C_{n} \hookrightarrow C_{b^{j} n} \hookrightarrow C_{n_{j}}$ of codes, whereas $C_{n}, C_{b^{j} n}, C_{n_{j}}$ are, respectively, primitive $\mathrm{BCH}$, non-primitive $\mathrm{BCH}$ and primitive $\mathrm{BCH}$ codes. Whereas the embeddings $C_{n} \hookrightarrow C_{b^{j} n}$ are defined as $a\left(x^{a}\right)=a_{0}+a_{1}\left(x^{a}\right)+\cdots+a_{n-1}\left(x^{a}\right)^{n-1} \mapsto a_{0}+a_{1}\left(x^{\frac{a}{b^{j}}}\right)^{b^{j}}+\cdots+$ $a_{n-1}\left(x^{\frac{a}{b^{j}}}\right)^{b^{j}(n-1)}=a\left(x^{\frac{a}{b^{j}}}\right)$, where $a\left(x^{a}\right) \in C_{n}$ and $a\left(x^{\frac{a}{b^{j}}}\right) \in C_{b^{j}}$. Also, if $g\left(x^{\frac{a}{b^{j}-1}}\right)$ is the generator polynomial of a binary non-primitive BCH code $C_{b^{j-1} n}^{j-1}$ in $\mathbb{F}_{2}\left[x ; \frac{a}{b^{j-1}} \mathbb{Z}_{0}\right]_{b^{j-1} n}$, then $g\left(x^{\frac{a}{b^{j}}}\right)$ is the generator polynomial of a binary non-primitive $\mathrm{BCH}$ code $C_{b^{j} n}$ in the monoid ring $\mathbb{F}_{2}\left[x ; \frac{a}{b^{j}} \mathbb{Z}_{0}\right]_{b^{j} n_{n}}$. Thus, a non-primitive $\mathrm{BCH}$ code $C_{b^{j-1} n}$ is embedded in a non-primitive $\mathrm{BCH}$ code $C_{b^{j}}$ under the monomorphism defined as $a\left(x^{\frac{a}{b^{j}-1}}\right) \mapsto a\left(x^{\frac{a}{b^{j}}}\right)$.

With the above discussion it follows the following theorem.

Theorem 3. [3, Theorem 6] Let $C_{n}$ be a primitive binary BCH code of length $n=2^{s}-1$ generated by a polynomial $g\left(x^{a}\right)$ of degree $r$ in $\mathbb{F}_{2}\left[x ; a \mathbb{Z}_{0}\right]$.

1. There exists a binary non-primitive $B C H$ code $C_{b^{j}}$ of length $b^{j} n$ generated by a polynomial $g\left(x^{\frac{a}{b^{j}}}\right)$ of degree $b^{j} r$ in $\mathbb{F}_{2}\left[x ; \frac{a}{b^{j}} \mathbb{Z}_{0}\right]$.

2. The binary primitive $\mathrm{BCH}$ code $C_{n}$ is embedded in a binary non-primitive $\mathrm{BCH}$ code $C_{b_{j} n_{n}}$, for each $j \geq 1$.

3. The binary $B C H$ codes of the sequence $\left\{C_{b^{j}}\right\}_{j \geq 1}$ have the following embedding

$$
C_{b n} \hookrightarrow C_{b^{2} n} \hookrightarrow \cdots \hookrightarrow C_{b^{j} n} \hookrightarrow \cdots
$$

Hence,

$$
\begin{aligned}
& \mathbb{F}_{2}\left[x ; a \mathbb{Z}_{0}\right] \quad \subset \quad \mathbb{F}_{2}\left[x ; \frac{a}{b} \mathbb{Z}_{0}\right] \quad \subset \quad \mathbb{F}_{2}\left[x ; \frac{a}{b^{2}} \mathbb{Z}_{0}\right] \quad \subset \quad \cdots \quad \mathbb{F}_{2}\left[x ; \frac{a}{b^{j}} \mathbb{Z}_{0}\right] \\
& \frac{\mathbb{F}_{2}\left[x ; a \mathbb{Z}_{0}\right]}{\left(\left(x^{a}\right)^{n}-1\right)} \simeq \frac{\mathbb{F}_{2}\left[x ; \frac{a}{b} \mathbb{Z}_{0}\right]}{\left(\left(x^{b}\right)^{b n}-1\right)} \simeq \frac{\mathbb{F}_{2}\left[x ; \frac{a}{b^{2}} \mathbb{Z}_{0}\right]}{\left(\left(x^{\frac{a}{b^{2}}}\right)^{b^{2} n}-1\right)} \bumpeq \ldots \frac{\mathbb{F}_{2}\left[x ; \frac{a}{b^{2}} \mathbb{Z}_{0}\right]}{\left(\left(x^{\frac{a}{b^{j}}}\right)^{b^{j}}-1\right)}
\end{aligned}
$$

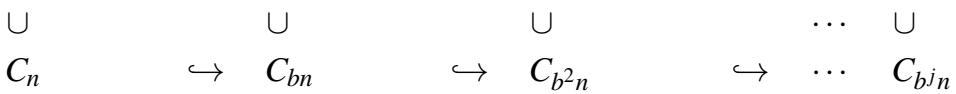

and a non-primitive $B C H$ code $C_{b^{j-1} n}$ is embedded in a non-primitive $B C H$ code $C_{b^{j} n}$ under the monomorphism defined by $a\left(x^{\frac{a}{b^{j}-1}}\right) \mapsto a\left(x^{\frac{a}{b^{j}}}\right)$.

Remark 4. The polynomial $g\left(x^{\frac{a}{b}}\right)$ can be obtained from $g\left(x^{\frac{a}{b^{j}}}\right)$ by substituting $x^{\frac{a}{b^{j}}}=y$ and replacing y by $y^{b^{j}-1}=x^{\frac{a}{b}}$.

Example 2.2. By [3, Example 2] and Example 2.1, it follows that the BCH codes with designed distance $d=2,3, \cdots, 9$ have generator polynomials $g\left(x^{\frac{2}{3}}\right), g\left(x^{\frac{2}{9}}\right)$ with the same minimum distance, error correction capability and code rate, where the differences are degree, data bits, code 
length and check sum of the code $C_{135}$ which is three times that of code $C_{45}$ and nine times of the code $C_{15}$. Similarly, on letting $\left(x^{\frac{2}{9}}\right)=y$, that is $x^{\frac{2}{3}}=y^{3}$, we get

$$
\begin{aligned}
g\left(x^{\frac{2}{9}}\right)= & \left(x^{\frac{2}{9}}\right)^{106}+\left(x^{\frac{2}{9}}\right)^{103}+\left(x^{\frac{2}{9}}\right)^{102}+\left(x^{\frac{2}{9}}\right)^{97}+\left(x^{\frac{2}{9}}\right)^{93}+\left(x^{\frac{2}{9}}\right)^{91}+\left(x^{\frac{2}{9}}\right)^{90}+ \\
& \left(x^{\frac{2}{9}}\right)^{61}+\left(x^{\frac{2}{9}}\right)^{58}+\left(x^{\frac{2}{9}}\right)^{57}+\left(x^{\frac{2}{9}}\right)^{52}+\left(x^{\frac{2}{9}}\right)^{48}+\left(x^{\frac{2}{9}}\right)^{46}+\left(x^{\frac{2}{9}}\right)^{45}+\left(x^{\frac{2}{9}}\right)^{16}+ \\
& \left(x^{\frac{2}{9}}\right)^{13}+\left(x^{\frac{2}{9}}\right)^{12}+\left(x^{\frac{2}{9}}\right)^{7}+\left(x^{\frac{2}{9}}\right)^{3}+\left(x^{\frac{2}{9}}\right)+1, \\
g(y)= & (y)^{106}+(y)^{103}+(y)^{102}+(y)^{97}+(y)^{93}+(y)^{91}+(y)^{90}+(y)^{61}+(y)^{58}+ \\
& (y)^{57}+(y)^{52}+(y)^{48}+(y)^{46}+(y)^{45}+(y)^{16}+(y)^{13}+(y)^{12}+(y)^{7}+ \\
& (y)^{3}+y+1, \\
g\left(y^{3}\right)= & \left(y^{3}\right)^{106}+\left(y^{3}\right)^{103}+\left(y^{3}\right)^{102}+\left(y^{3}\right)^{97}+\left(y^{3}\right)^{93}+\left(y^{3}\right)^{91}+\left(y^{3}\right)^{90}+\left(y^{3}\right)^{61}+ \\
& \left(y^{3}\right)^{58}+\left(y^{3}\right)^{57}+\left(y^{3}\right)^{52}+\left(y^{3}\right)^{48}+\left(y^{3}\right)^{46}+\left(y^{3}\right)^{45}+\left(y^{3}\right)^{16}+\left(y^{3}\right)^{13}+ \\
& \left(y^{3}\right)^{12}+\left(y^{3}\right)^{7}+\left(y^{3}\right)^{3}+\left(y^{3}\right)+1, \\
g\left(x^{\frac{2}{3}}\right)= & \left(x^{\frac{2}{3}}\right)^{106}+\left(x^{\frac{2}{3}}\right)^{103}+\left(x^{\frac{2}{3}}\right)^{102}+\left(x^{\frac{2}{3}}\right)^{97}+\left(x^{\frac{2}{3}}\right)^{93}+\left(x^{\frac{2}{3}}\right)^{91}+\left(x^{\frac{2}{3}}\right)^{90}+\left(x^{\frac{2}{3}}\right)^{61} \\
& +\left(x^{\frac{2}{3}}\right)^{58}+\left(x^{\frac{2}{3}}\right)^{57}+\left(x^{\frac{2}{3}}\right)^{52}+\left(x^{\frac{2}{3}}\right)^{48}+\left(x^{\frac{2}{3}}\right)^{46}+\left(x^{\frac{2}{3}}\right)^{45}+\left(x^{\frac{2}{3}}\right)^{16}+\left(x^{\frac{2}{3}}\right)^{13} \\
& +\left(x^{\frac{2}{3}}\right)^{12}+\left(x^{\frac{2}{3}}\right)^{7}+\left(x^{\frac{2}{3}}\right)^{3}+\left(x^{\frac{2}{3}}\right)+1 \\
& \left(x^{\frac{2}{3}}\right)^{16}+\left(x^{\frac{2}{3}}\right)^{13}+\left(x^{\frac{2}{3}}\right)^{12}+\left(x^{\frac{2}{3}}\right)^{7}+\left(x^{\frac{2}{3}}\right)^{3}+\left(x^{\frac{2}{3}}\right)+1 \in \mathbb{F}_{2}\left[x ; \frac{2}{3} \mathbb{Z}_{0}\right]_{45} .
\end{aligned}
$$

Similarly, for

$$
\begin{aligned}
g\left(x^{\frac{2}{9}}\right)= & \left(x^{\frac{2}{9}}\right)^{112}+\left(x^{\frac{2}{9}}\right)^{108}+\left(x^{\frac{2}{9}}\right)^{105}+\left(x^{\frac{2}{9}}\right)^{102}+\left(x^{\frac{2}{9}}\right)^{100}+\left(x^{\frac{2}{9}}\right)^{99}+\left(x^{\frac{2}{9}}\right)^{94}+ \\
& \left(x^{\frac{2}{9}}\right)^{91}+\left(x^{\frac{2}{9}}\right)^{90}+\left(x^{\frac{2}{9}}\right)^{67}+\left(x^{\frac{2}{9}}\right)^{63}+\left(x^{\frac{2}{9}}\right)^{60}+\left(x^{\frac{2}{9}}\right)^{57}+\left(x^{\frac{2}{9}}\right)^{55}+ \\
& \left(x^{\frac{2}{9}}\right)^{54}+\left(x^{\frac{2}{9}}\right)^{49}+\left(x^{\frac{2}{9}}\right)^{46}+\left(x^{\frac{2}{9}}\right)^{45}+\left(x^{\frac{2}{9}}\right)^{22}+\left(x^{\frac{2}{9}}\right)^{18}+\left(x^{\frac{2}{9}}\right)^{15}+ \\
& \left(x^{\frac{2}{9}}\right)^{12}+\left(x^{\frac{2}{9}}\right)^{10}+\left(x^{\frac{2}{9}}\right)^{9}+\left(x^{\frac{2}{9}}\right)^{4}+\left(x^{\frac{2}{9}}\right)+1,
\end{aligned}
$$

it follows that

$$
g\left(x^{\frac{2}{3}}\right)=\left(x^{\frac{2}{3}}\right)^{22}+\left(x^{\frac{2}{3}}\right)^{18}+\left(x^{\frac{2}{3}}\right)^{15}+\left(x^{\frac{2}{3}}\right)^{12}+\left(x^{\frac{2}{3}}\right)^{10}+\left(x^{\frac{2}{3}}\right)^{9}+\left(x^{\frac{2}{3}}\right)^{4}+\left(x^{\frac{2}{3}}\right)+1,
$$

which is the generating polynomial of a BCH code $(45,23)$ having design distance $d_{2}=6,7$. In this way. we can obtain a non-primitive binary $B C H$ code $C_{15}$ from a non-primitive binary $B C H$ code $C_{45}$. On writing the corresponding code vectors of the generating polynomials

$$
\begin{aligned}
g\left(x^{\frac{2}{3}}\right)= & \left(x^{\frac{2}{3}}\right)^{16}+\left(x^{\frac{2}{3}}\right)^{13}+\left(x^{\frac{2}{3}}\right)^{12}+\left(x^{\frac{2}{3}}\right)^{7}+\left(x^{\frac{2}{3}}\right)^{3}+\left(x^{\frac{2}{3}}\right)+1 \\
g\left(x^{\frac{2}{9}}\right)= & \left(x^{\frac{2}{9}}\right)^{106}+\left(x^{\frac{2}{9}}\right)^{103}+\left(x^{\frac{2}{9}}\right)^{102}+\left(x^{\frac{2}{9}}\right)^{97}+\left(x^{\frac{2}{9}}\right)^{93}+\left(x^{\frac{2}{9}}\right)^{91}+ \\
& \left(x^{\frac{2}{9}}\right)^{90}+\left(x^{\frac{2}{9}}\right)^{61}+\left(x^{\frac{2}{9}}\right)^{58}+\left(x^{\frac{2}{9}}\right)^{57}+\left(x^{\frac{2}{9}}\right)^{52}+\left(x^{\frac{2}{9}}\right)^{48}+\left(x^{\frac{2}{9}}\right)^{46}+ \\
& \left(x^{\frac{2}{9}}\right)^{45}+\left(x^{\frac{2}{9}}\right)^{16}+\left(x^{\frac{2}{9}}\right)^{13}+\left(x^{\frac{2}{9}}\right)^{12}+\left(x^{\frac{2}{9}}\right)^{7}+\left(x^{\frac{2}{9}}\right)^{3}+\left(x^{\frac{2}{9}}\right)+1,
\end{aligned}
$$

it follows that

$$
\begin{aligned}
v^{1}= & (11010001000011001) \\
v^{2}= & (11010001000011001000000000000000000000000000011010001 \\
& 000011001000000000000000000000000000011010001000011001)
\end{aligned}
$$


The binary bits of $v^{1}$ are properly overlapped on the bits of $v^{2}$, in fact, they are repeated three times after a particular pattern. Hence, the generating matrix $G_{2}$ of $g\left(x^{\frac{2}{9}}\right)$ contains the generating matrix $G_{1}$ of $g\left(x^{\frac{2}{3}}\right)$ such that $G_{2}=\oplus_{1}^{3} G_{1}$.

\section{ALGORITHM}

In this section, we propose an algorithm to calculate a non-primitive $\mathrm{BCH}$ code of length $b^{j} n$ using a primitive $\mathrm{BCH}$ code of length $n$, where the encoding and decoding of the code are carried out in Matlab. The process of developing algorithm is divided into two major steps, i.e., encoding and decoding of a non-primitive BCH code of length $b^{j} n$.

\subsection{Encoding of a non-primitive BCH code of length $b^{j} n$.}

In encoding, we first calculate a primitive polynomial of degree $s$ by invoking Matlab's built in command "bchgenpoly". After this operation, a non-primitive polynomial of degree $b s$ is calculated. With the help of a root, say $\alpha^{\prime}$, the elements of the Galois field $G F\left(2^{b n}\right)$ are calculated such that $\left(\alpha^{\prime}\right)^{b^{j} n}=1$ and after we obtain the minimal polynomials of these elements. Finally, we get a non-primitive polynomial with the help of these minimal polynomials. Thus, these design distances are calculated through which the number of errors that can be corrected in each $\mathrm{BCH}$ code are determined. Some methods are developed in order to achieve a specified result. In Table 3 , we show a list of these methods and its description.

Table 3: Encoding modulation description.

\begin{tabular}{llll}
\hline & Module & Input & Output \\
\hline $\mathrm{a}$ & Elements of Galois Field & $b * n$ & $\alpha^{\prime}[i]$ \\
$\mathrm{b}$ & Bchgenpoly & $n, k$ & $g[i]$ \\
$\mathrm{c}$ & Cyclotomic_cosets & $\alpha^{\prime}[i], b, k$ & $c[i]$ \\
$\mathrm{d}$ & Design_distance & $c[i]$ & $d$ \\
$\mathrm{e}$ & error & $d$ & $t$ \\
\hline
\end{tabular}

The steps of the algorithm are explained as follows:

a. Elements of Galois field (alpha array) $\alpha^{\prime}[i]$ ).

This step calculates all the elements of the Galois field $G F\left(2^{b n}\right)$ using a root, say $\alpha^{\prime}$, of a nonprimitive polynomial of degree $b s$ such that $\left(\alpha^{\prime}\right)^{b n}=1$. The element $\alpha^{\prime}$ is called of alpha array and denoted by $\alpha^{\prime}[i]$. Now, denote the index array by $A[$ index $]$. For a given input $b * n$, the nonprimitive polynomial of degree $b s$ gives the first element of the array $\alpha^{\prime}[i]$. By increasing its power, each element of the array $\alpha^{\prime}[i]$ is calculated in outer loop. Then in nested while loop their corresponding values are determined in such a way that if the value of any element in the array exceed $b s$, we take the remainder rem(index, $b s$ ) (step 9 of the algorithm). The loop breaks when the condition for identity is met. 


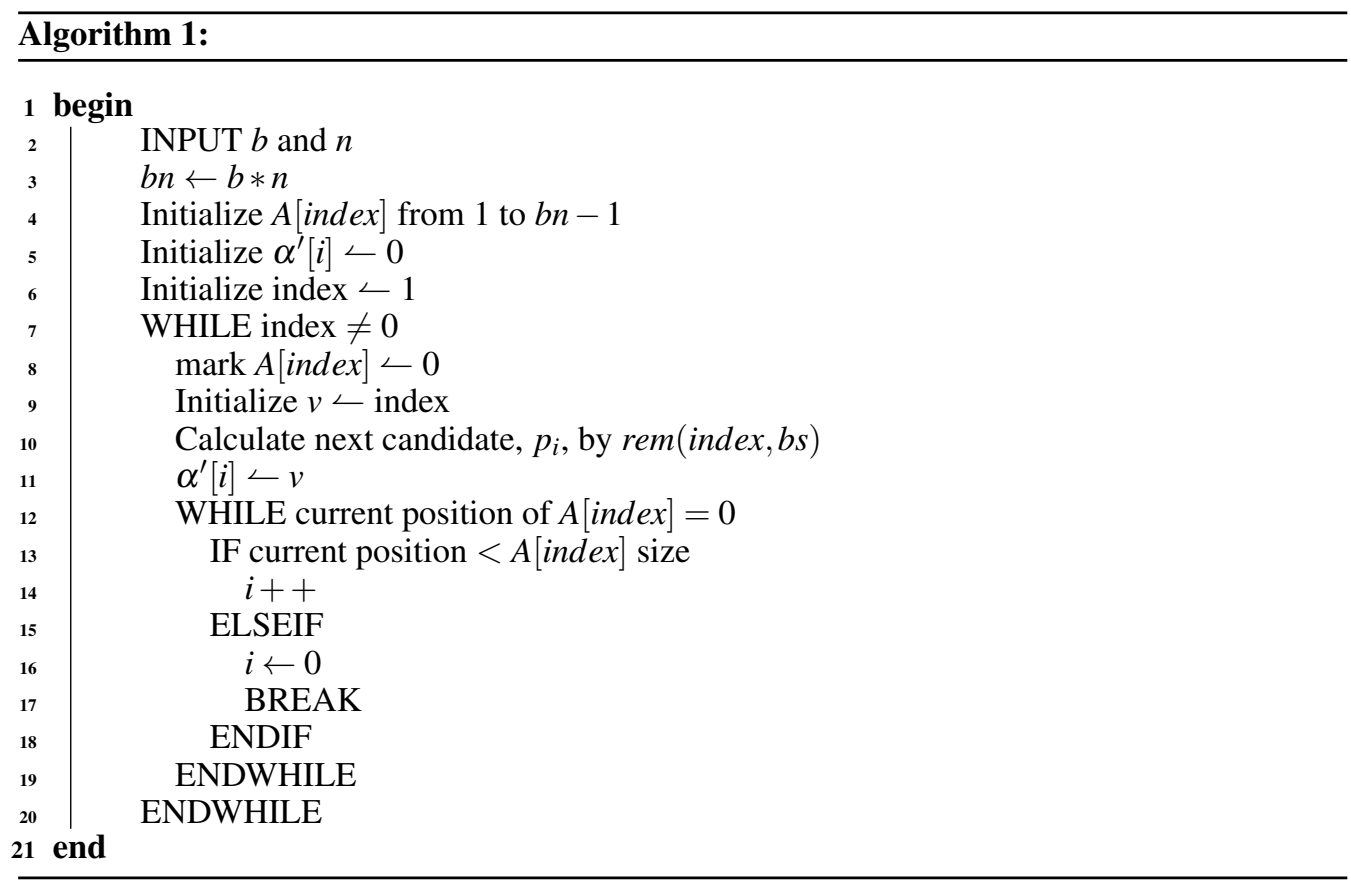

\section{b. BCH generator polynomial $(g[i])$.}

The Matlab builds a function and its complete documentation is found under http://www.mathworks.com/help/comm/ref/bchgenpoly.html. In this module, we get the generator polynomial of primitive $\mathrm{BCH}$ code of length $n$.

1 INPUT $n$ and $k$

2 OUTPUT $g[i]$

c. Cyclotomic cosets $(c[i])$.

Given $\alpha^{\prime}[i]$, dimension of code $k$ and positive integer $b$, cyclotomic cosets $c[i]$ are calculated. Length of $c[i]$ is initialized to at max $b * k$, in short all elements should not exceed the max length. Loop start from 2 to max length and calculate unique values in given $\alpha^{\prime}[i]$. The process stops when we get sum of two elements $=2$. Once cyclotomic cosets are calculated, we can calculate the minimal polynomials for $\mathrm{BCH}$ codes.

\section{d. Non-primitive generating polynomial $g^{\prime}[i]$.}

Given primitive polynomial $p[i]$ and $b$. First find the highest degree of $p[i]$ i.e., $s$ and initial degree of non-primitive polynomial $p^{\prime}[i]$ i.e., $b s$. Initialize each element of $p^{\prime}[i]$ of length $b s$ to 0 . Iterate loop from 2 to $b s$ in order to initialize coefficient array to 1. Finally, iterate each element if coeft_array and modify the value if $p^{\prime}[i]$ at position $i$ to the value of coeft_array at $i$. Finally insert 1 at position 0 of $p^{\prime}[i]$, i.e., when its first element becomes 0 . The output of this module 


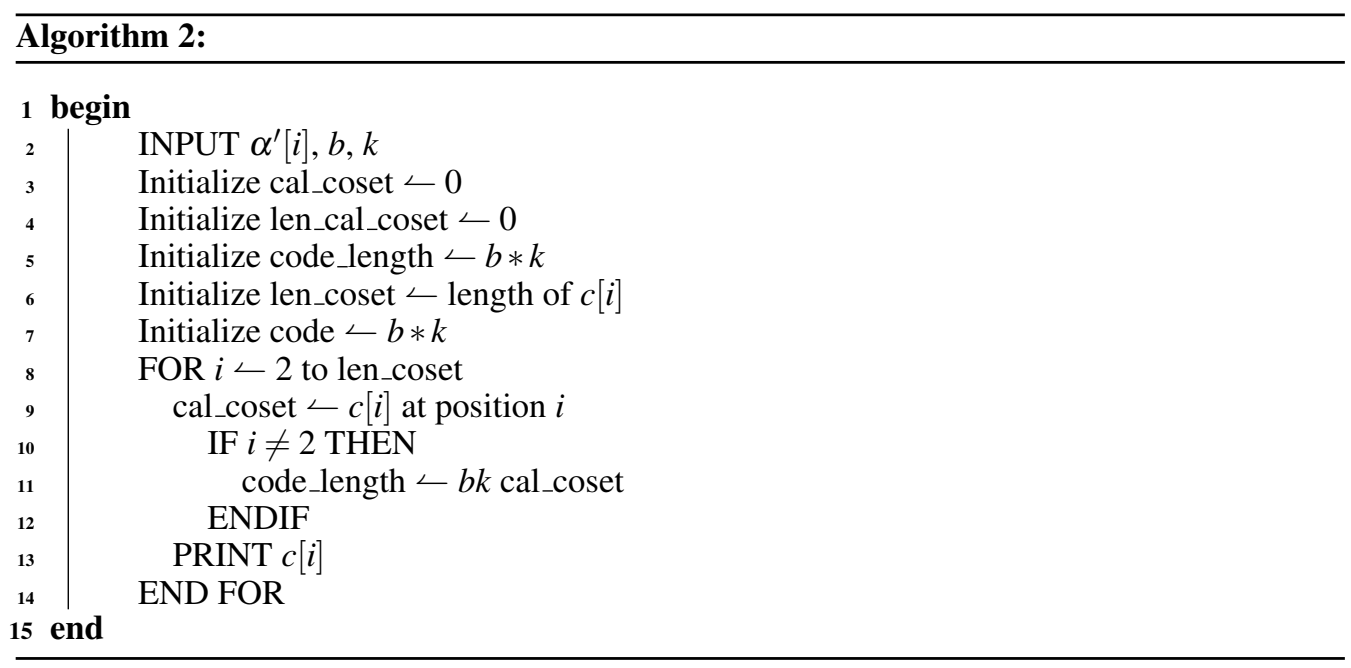

play an integral role for calculating non-primitive $\mathrm{BCH}$ generating polynomial. It is denoted by $g^{\prime}$ in our algorithm. We are interested in rows of obtained matrix. Using the matrix we obtained, the code for non-primitive generating polynomial of length $b$. Finally these values are printed out and saved in file for further usage.

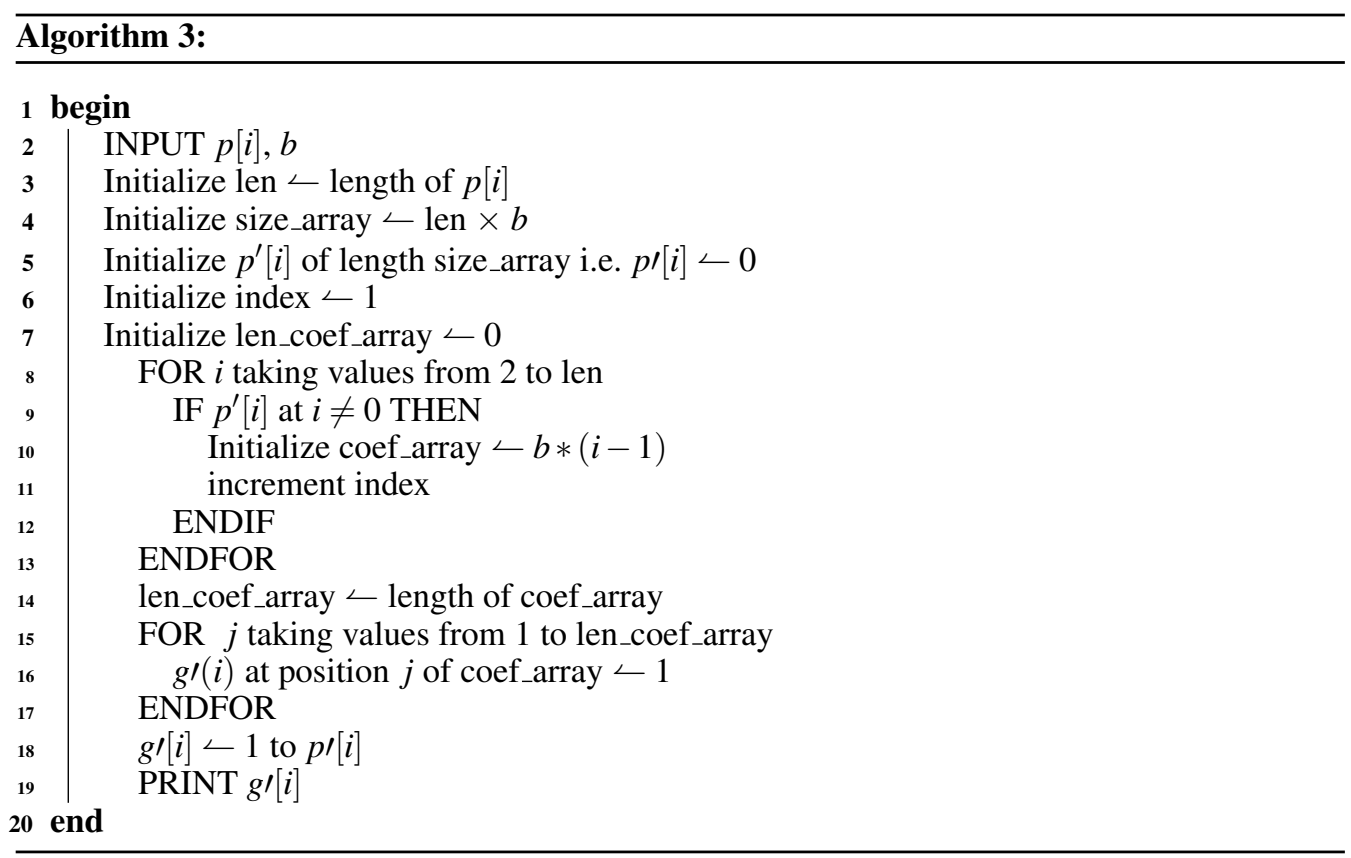




\section{e. Designed distance $(d)$.}

Here design distance is calculated from $g^{\prime}[i]$. The length of coset array $c l$ is determined and then iterate index from 2 to $c l$, calculate next index $n i$ by increment current index. If $n i \leq c l$, then next element of coset is calculated to the value of coset array at position of next index. Otherwise the $b n$ is assigned to next coset. The whole process iterates to lencoset coset and finally stops at the last coset. Design distance $d$ is calculated from the last value of coset array at position 1 .

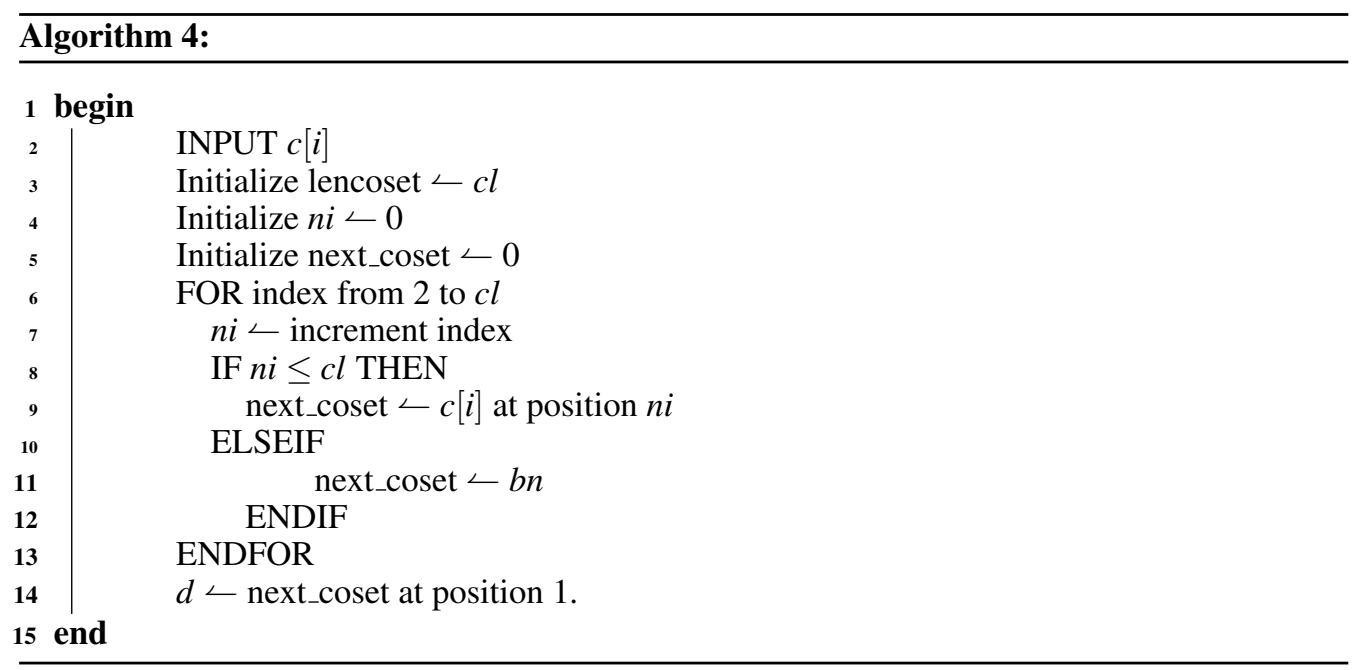

\section{f. Error correction capability.}

For given designed distance $d$, the error correction capability of a code $t$ is calculated.

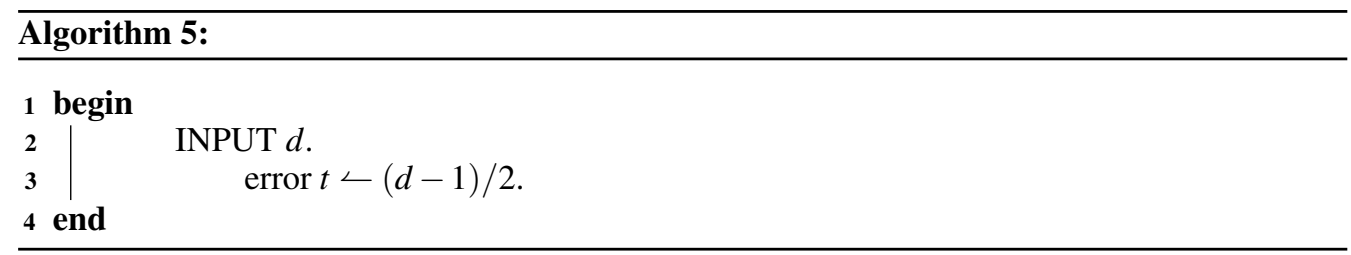

\subsection{Error correction in received polynomial (decoding)}

In decoding step for a received polynomial, we first calculate the syndrome matrix $S[i]$. Then the D_matrix is calculated that should be invertible. After this error locator polynomial is determined whose roots give the exact position of errors in the received polynomial. Finally the received polynomial is corrected. To find the error vector and obtain the corrected codeword following scheme is used. Table 4 shows list of the following steps for error correction. 
Table 4: Encoding modulation description.

\begin{tabular}{llll}
\hline & Module & Input & Output \\
\hline a & Syndrome_Matrix & $d, b n, b k, \alpha^{\prime}, r[i]$ & $S^{\prime}[i]$ \\
b & Calculate $D \_m a t r i x$ & $t, S^{\prime}[i]$ & $D \_$matrix \\
c & Is $D$ invertible & $t, D \_$matrix & $|D| \neq 0$ \\
d & error_locator_poly & $t, D \_m a t r i x, S^{\prime}[i]$ & $f[i]$ \\
e & error_position & $f[i]$ & $e[i]$ \\
f & error_values & $t, S[i], D \_$matrix, $e[i], b n, p m[i]$ & $e v[i]$ \\
h & Correct_recieved & $t, b n, e[i], e v[i], r[i], \alpha^{\prime}$ & $v[i]$ \\
& polynomial & & \\
\hline
\end{tabular}

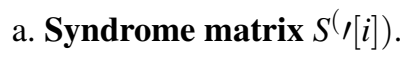

Given design distance $d, b n$, message length $b k$ and received polynomial $r[i]$, syndrome matrix $S^{\prime}[i]$ can be calculated. The length of $S^{\prime}[i]$ is initialize to the difference of $b n$ and $b k$, i.e., $b n-b k$. Furthermore, $S^{\prime}[i]$ is initialized by element of Galois field at position $i$ i.e. $G F[i]$. Nested loop are used to calculate $S^{\prime}[i]$. Upper loop is limited to the length of $b n+d-2$, where $S^{\prime}[i]$ equalizes to power of $\alpha^{\prime}$ and in nested loop $S^{\prime}[i]$ equalize to power of $S^{\prime}[i]$ and the iterator. Once the values of $S^{\prime}[i]$ are filled with the above values of $S^{\prime}[i]$, it follows that these $S^{\prime}[i]$ can be calculated as product of $r[i]$ and $\left(S^{\prime}[i]\right)^{t}$.

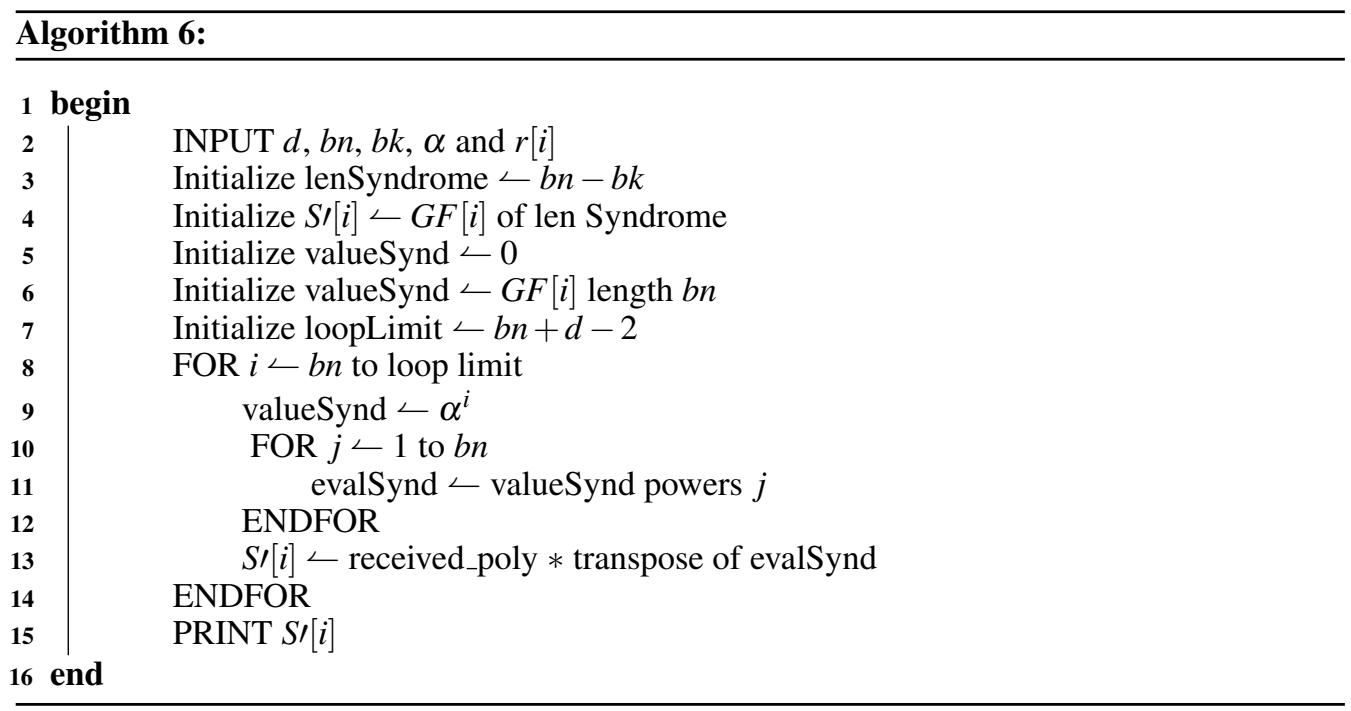

\section{b. $D$ matrix $(D[i])$.}

Given error $t$ and $S^{\prime}[i], D$ matrix is calculated and then double loops operation on syndrome matrix is carried out and suitable values from syndrome matrix is scanned out. The process is as 
follows: calculate $G F[i]$ of length $t$ and $D$-matrix is initialized to that value. $D[i]$ in nested loop for loops. Both loops iterates from 1 to $t . D[i]$ values are $S^{\prime}[i]$ values at position $i$ of sum of loops iterators to 1 .

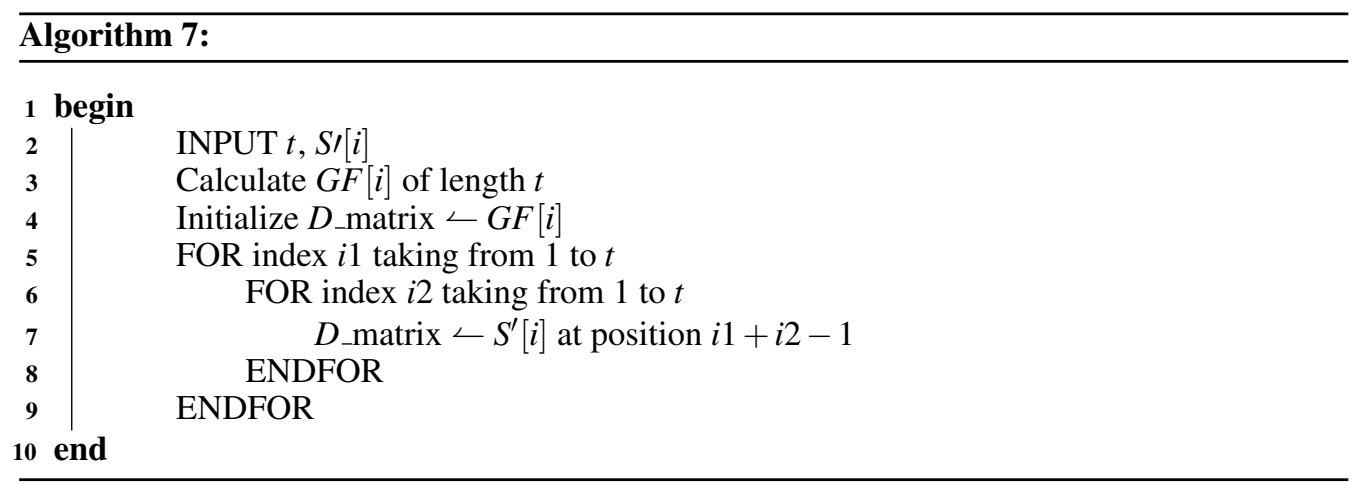

\section{c. Is $D$ invertible.}

This module check if $D[i]$ is invertible. If $D[i]$ is invertible, then the algorithm works, otherwise error $t$ is decremented and $D$ matrix is again calculated. These operations are carried out till error $t$ becomes 0 . If $t$ becomes 0 , then algorithm will exit i.e panic condition occurs as in step 10 .

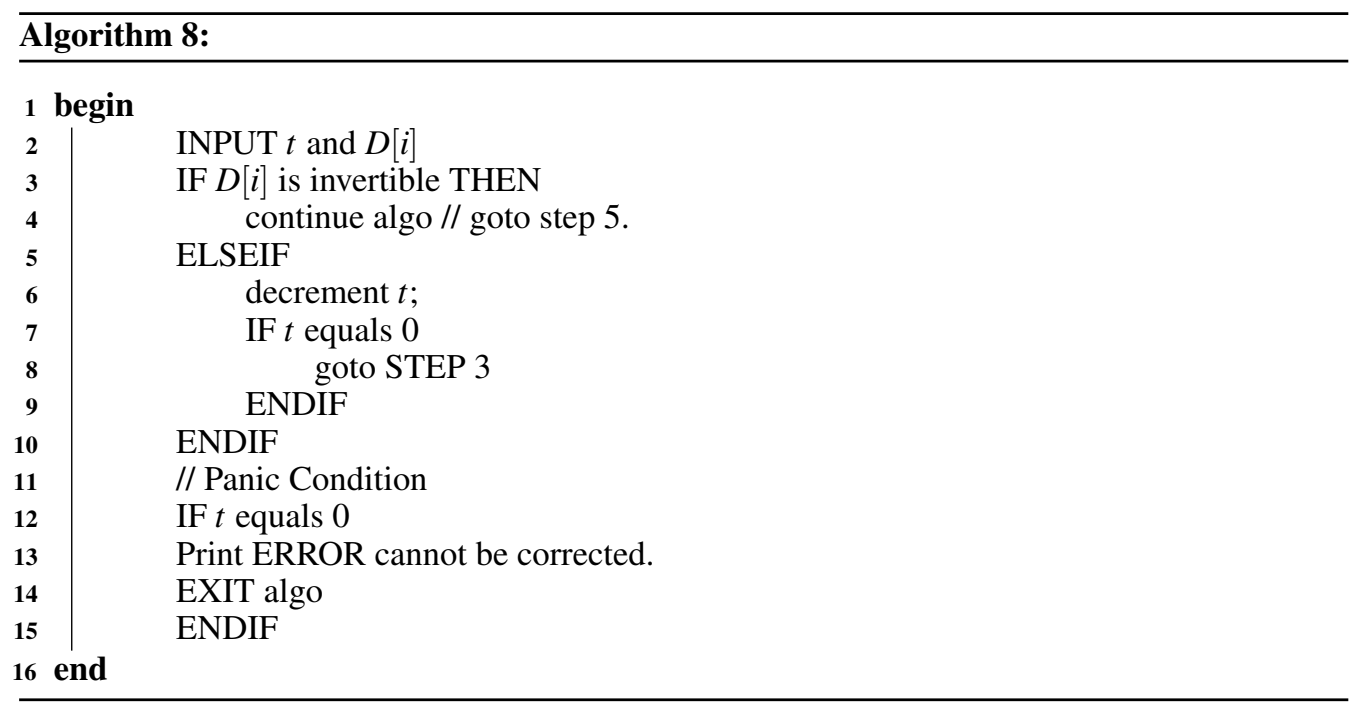

\section{d. Error locator polynomial $(\mathbf{f}[\mathbf{i}])$.}

Given input $t, D[i]$ and $S^{\prime}[i]$, error locator polynomial $f[i]$ is calculated. First, initialize product matrix $p m[i]$ and $f[i]$ to $\alpha^{\prime t}[i]$. Then, iterate the loop from 1 to $t, p m[i]$ is filled with the value of $S^{\prime}[i]$ at $t+i$. After the loops ends, the value of $\left(S^{\prime}[i] * p m[i]\right)^{-1}$ get equals to temporary matrix 
$T^{\prime \prime}[i]$. Once the temporary matrix $T^{\prime \prime}[i]$ is achieved $f[i]$ is taken as the transpose of that temporary matrix $\left(T^{\prime \prime}[i]\right)^{t}$ discuss in step 10 .

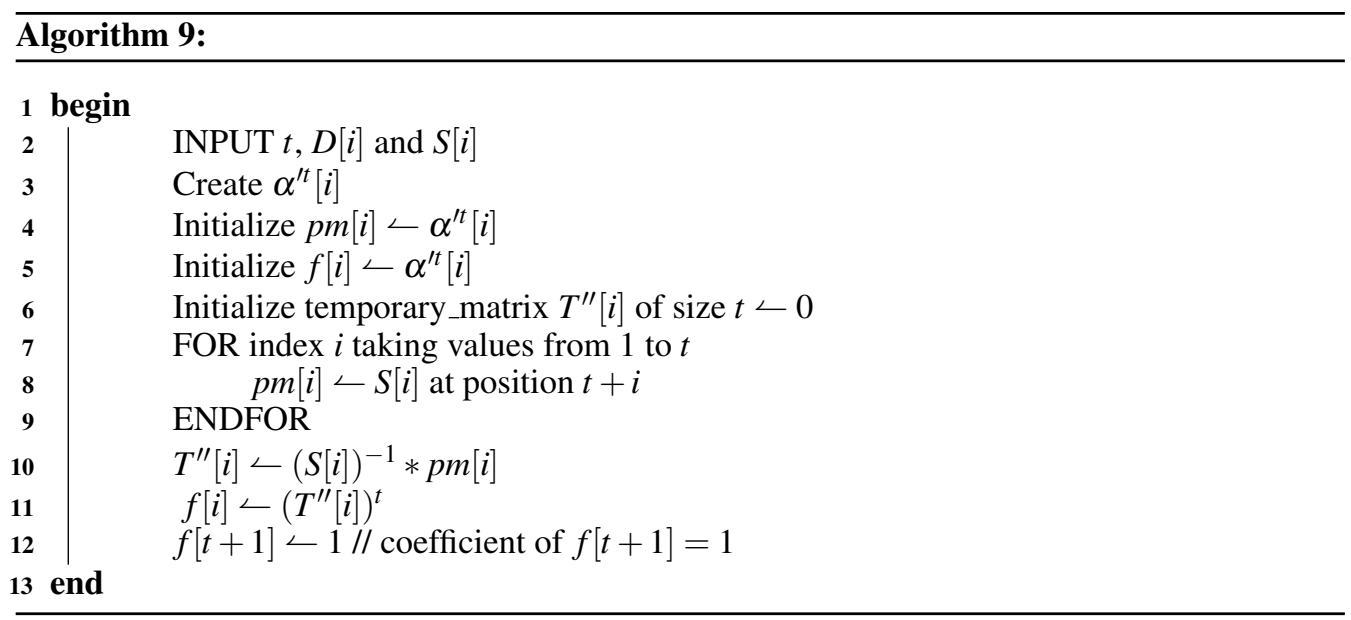

\section{e. Error position matrix $(e[i])$.}

Based on $f[i]$ we can determine error position. First, the roots of $f[i]$ is calculated and then we take its inverse. The values we obtain are in matrix form and these manifest error position.

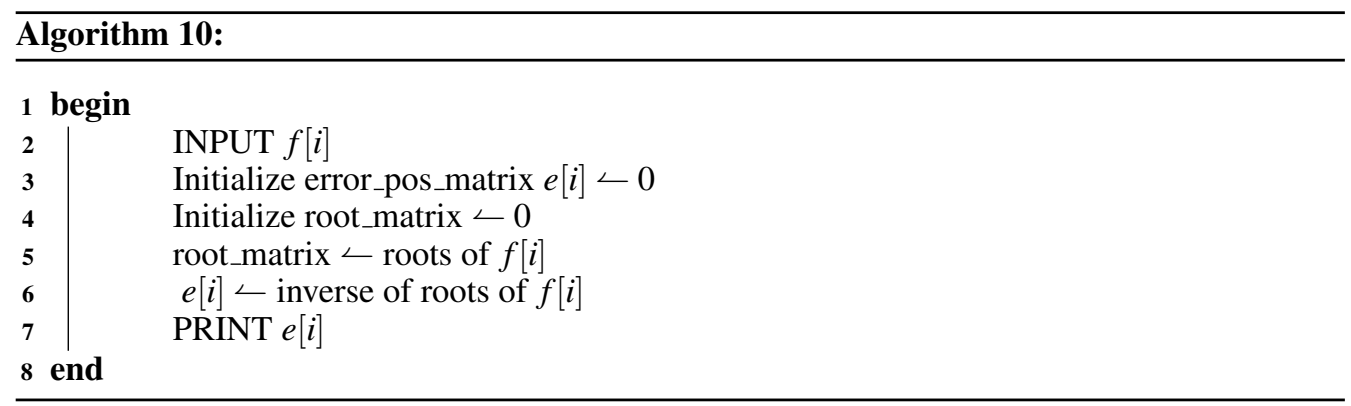

\section{f. Error values $(e v[i])$.}

Once error position $e[i]$ is determined we can easily calculate their respective values. The nested for loops are used to determine error values. Both of the loops iterate from 1 to $t$, in the first loop values from $D \_$matrix can be taken while in the next loop value of product matrix $p m[i]$ can be taken along with $S^{\prime}[i]$. Finally, $e v[i]$ get equated to $\left(D \_ \text {matrix } * p m[i]\right)^{-1}$ as discusses on line 9 .

g. Correct received polynomial $r[i]$.

Once we have calculated error positions $e[i]$ and error values $e v[i]$ the received polynomial $r[i]$ can be corrected. Here input parameters are $e[i], e v[i], r[i]$ and $b n$. First estimated codeword denoted by est_code is calculated using various operations i.e., taking loops to $t, b n$ and power of 


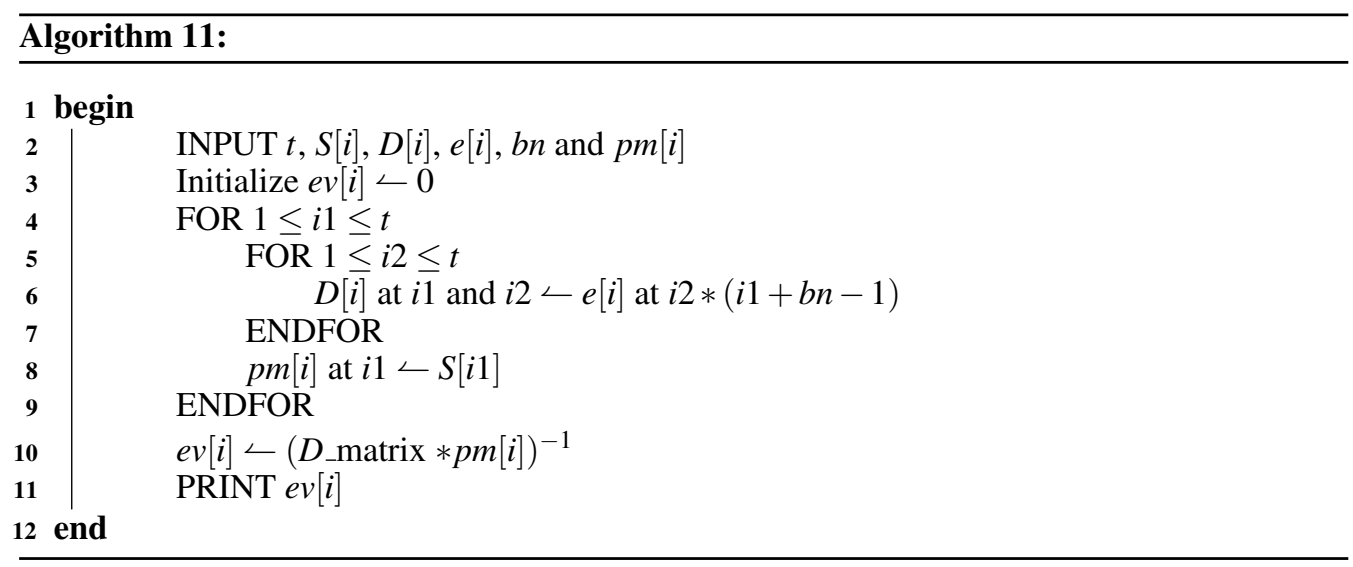

Galois field. The received polynomial is corrected by subtracting error polynomial and we get the corrected codeword $v[i]$.

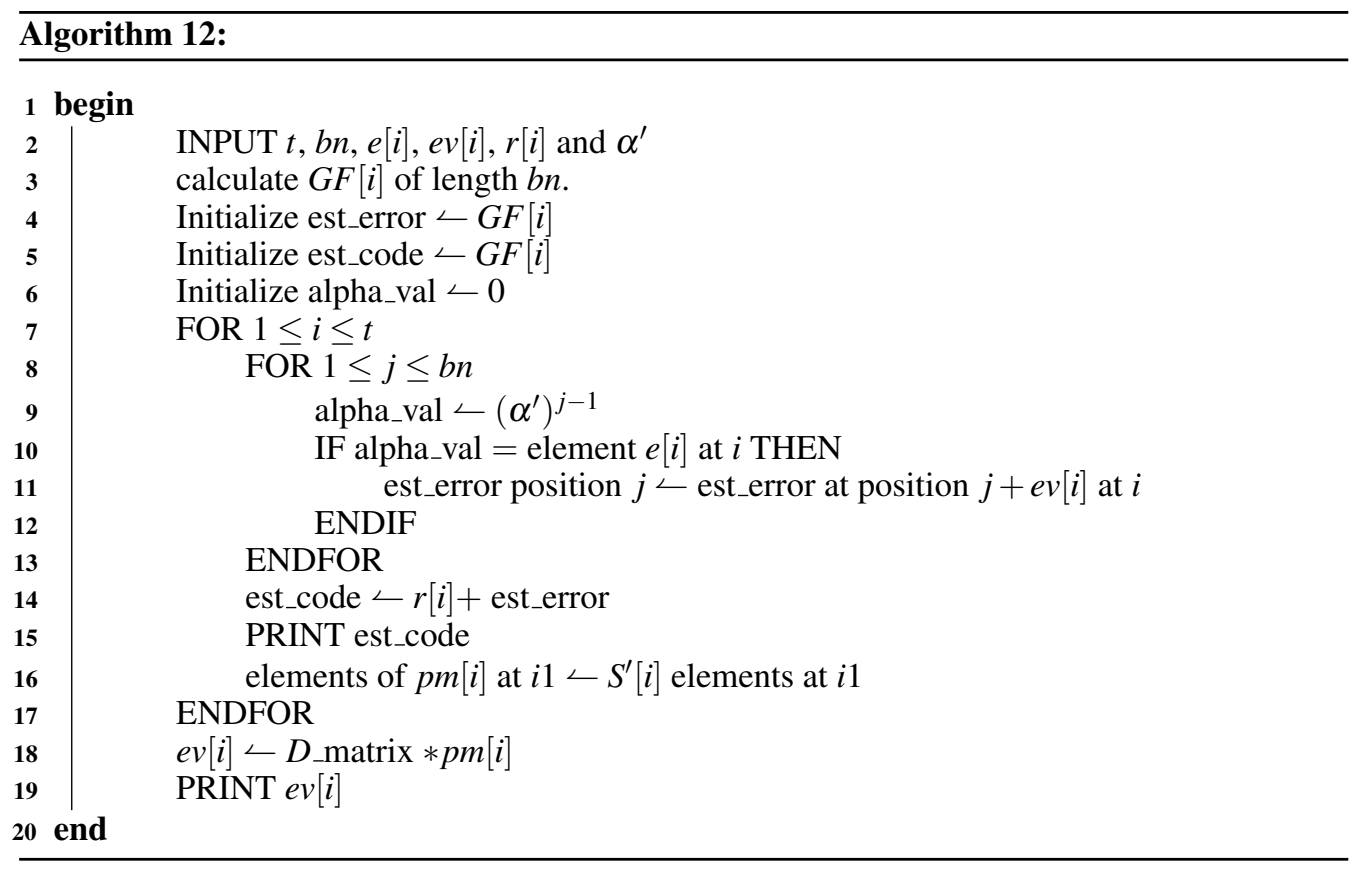

Example 3.1. For the code of length 45 simulation is carried out as follows: in this case $b=3$ and $n=15$. Using $n=15$ and $k=11$, Matlab's build in function genpoly is invoked in order to find primitive polynomial, i.e., $p(i)=x^{4}+x+1$, as explain in Table 3. With $b=3$ and $p(i)=$ $x^{4}+x+1$, non primitive poly function is invoked, as described in Table 3 step 4, here nonprimitive polynomial named as $p^{\prime}(i)$ is obtained. Output for $p^{\prime}(i)$ is $x^{12}+x^{9}+1$. Cyclotomic cosets, i.e., coset_array values are also calculated. First elements of Galois field in step 1 of 
Table 1, is invoked to find the power of alpha till $\alpha^{45}=1$. With coset_array in hand, the designed distance $d$ can be calculated, which is the first element of next coset_array. Last but not the least errort is calculated against the given designed distance $d$. Code rate $R$ is also calculated against each $k_{1}$ and bn but is not mentioned in previous section. The output are as follows: Cyclotomic

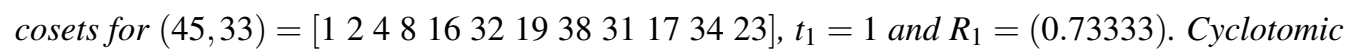

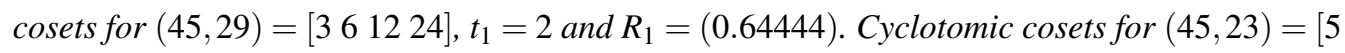

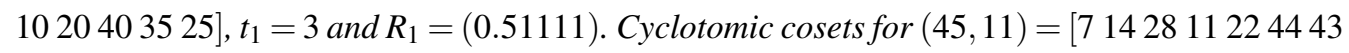
$4137291326], t_{1}=4$ and $R_{1}=(0.24444)$. Cyclotomic cosets for $(45,5)=[1530], t_{1}=10$ and $R_{1}=(0.11111)$. Now comes error correction in received polynomial. In this the code $(45,29)$ is taken under consideration, with designed distance $d_{1}=5$ and $t_{1}=2$. Let the received polynomial be

$$
x^{44}+x^{16}+x^{13}+x^{12}+x^{11}+x^{7}+x^{3}+x+1 .
$$

With the given values $d_{1}, k_{1}$, and received polynomial, syndrome_matrix is calculated. The output for syndromes are $S_{1}=\alpha^{2}, S_{2}=\alpha^{4}, S_{3}=\alpha^{30}, S_{4}=\alpha^{8}$. Next, we arrange syndrome values in linear equation form that is $A x=B$. Where $A=\left[S_{1}, S_{2} ; S_{2}, S_{3}\right]$ and $B=\left[S_{3}, S_{4}\right]$. Matrix $A$ is named as $t$ matrix of $t \times t$ dimension. Thus, we find the whether the $t \_m a t r i x$ is singular or not. If the determinant of $t$ matrix is nonzero then error locator polynomial is calculated. Next error position is calculated from sigma_matrix which is obtained from the coefficients of error locator polynomial. For the given values, error_positions are 44 and 11 . Hence the error polynomial is $x^{44}+x^{11}$. On subtracting error polynomial from received polynomial the following code polynomial

$$
x^{16}+x^{13}+x^{12}+x^{7}+x^{3}+x+1
$$

is obtained. All of the above equations are obtained by using Matlab symbolic toolbox.

With the help of the above discussed algorithm many examples on non-primitive BCH codes of length $b n, b^{2} n, b^{3} n$ are constructed corresponding to primitive $\mathrm{BCH}$ code of length $n$. The parameters for all binary non-primitive $\mathrm{BCH}$ codes of length $b n, b^{2} n, b^{3} n$, where $n \leq 2^{6}-1$ and $b$ is either 3 or 7 are given in Table 5 .

Table 5 manifests the error and code rate values against some selected codes which we have obtained after simulating our algorithm. These codes are of length $b n, b^{2} n$ and $b^{3} n$, where $n \leq$ $2^{6}-1$, and $b=3,7 . k_{1}, k_{2}$ and $k_{3}$ are dimensions of the codes $C_{b n}, C_{b^{2} n}$ and $C_{b^{3} n}$, respectively.

\section{Interleaved Codes}

From Table 5, it is observed that corresponding to a primitive $(n, k)$ code there are $(b n, b k)$, $\left(b^{2} n, b^{2} k_{2}\right),\left(b^{3} n, b^{3} k\right)$ codes with same error correction capability and code rate. These codes are found to be interleaved codes (Interleaving is a periodic and reversible reordering of codes of $L$ transmitted bits) of depth $b, b^{2}$ and $b^{3}$. Hence along with random error correction capability these codes can correct burst of error of length $b, b^{2}$ and $b^{3}$, respectively. The term burst of error means that two or more bits in the received word has changed from 1 to 0 or from 0 to 1 . The length of the burst is measured from the first corrupted bit to the last corrupted bit. Similarly, for the code $(b n, b k)$ the codes $\left(b^{2} n, b^{2} k\right)$ and $\left(b^{3} n, b^{3} k\right)$ are interleaved codes of depth $b^{2}$ and $b^{3}$, 
Table 5: Parameters of binary non-primitive $\mathrm{BCH}$ codes.

\begin{tabular}{|c|c|c|c|c|c|c|c|c|c|c|c|c|}
\hline n & bn & $\mathbf{k}_{1}$ & $\mathbf{t}_{1}$ & $\mathbf{R}_{1}$ & $\mathbf{b}^{2} \mathbf{n}$ & $\mathbf{k}_{2}$ & $\mathbf{t}_{2}$ & $\mathbf{R}_{2}$ & $\mathbf{b}^{3} \mathbf{n}$ & $\mathbf{k}_{3}$ & $\mathbf{t}_{3}$ & $\mathbf{R}_{3}$ \\
\hline \multirow[t]{13}{*}{15} & 45 & 33 & 1 & 0.733 & 135 & 99 & 1 & 0.733 & 405 & 297 & 1 & 0.733 \\
\hline & & 29 & 2 & 0.644 & & 87 & 2 & 0.644 & & 261 & 2 & 0.644 \\
\hline & & 23 & 3 & 0.511 & & 69 & 3 & 0.511 & & 207 & 3 & 0.511 \\
\hline & & 11 & 4 & 0.244 & & 33 & 4 & 0.244 & & 99 & 4 & 0.244 \\
\hline & & 7 & 7 & 0.155 & & 29 & 7 & 0.214 & & 87 & 7 & 0.214 \\
\hline & & 5 & 10 & 0.111 & & 23 & 10 & 0.170 & & 69 & 10 & 0.170 \\
\hline & & 1 & 22 & 0.022 & & 11 & 13 & 0.081 & & 33 & 13 & 0.081 \\
\hline & & & & & & 7 & 22 & 0.051 & & 29 & 22 & 0.071 \\
\hline & & & & & & 5 & 31 & 0.037 & & 23 & 31 & 0.056 \\
\hline & & & & & & 1 & 67 & 0.007 & & 11 & 40 & 0.027 \\
\hline & & & & & & & & & & 7 & 67 & 0.017 \\
\hline & & & & & & & & & & 5 & 94 & 0.012 \\
\hline & & & & & & & & & & 1 & 202 & 0.002 \\
\hline \multirow[t]{8}{*}{63} & 189 & 171 & 1 & 0.904 & 567 & 513 & 1 & 0.904 & 1701 & 1539 & 1 & 0.904 \\
\hline & & 165 & 2 & 0.873 & & 495 & 2 & 0.873 & & 1485 & 2 & 0.873 \\
\hline & & 147 & 3 & 0.777 & & 441 & 3 & 0.777 & & 1323 & 3 & 0.777 \\
\hline & & 129 & 4 & 0.682 & & 387 & 4 & 0.682 & & 1161 & 4 & 0.682 \\
\hline & & 123 & 5 & 0.650 & & 381 & 5 & 0.672 & & 1143 & 5 & 0.671 \\
\hline & & 105 & 6 & 0.555 & & 327 & 6 & 0.576 & & 981 & 6 & 0.576 \\
\hline & & 87 & 7 & 0.460 & & 273 & 7 & 0.481 & & 819 & 7 & 0.481 \\
\hline & & 81 & 10 & 0.428 & & 255 & 10 & 0.449 & & 765 & 10 & 0.449 \\
\hline \multirow[t]{21}{*}{63} & 189 & 75 & 11 & 0.3968 & 567 & 237 & 11 & 0.4179 & 1701 & 711 & 11 & 0.417 \\
\hline & & 57 & 13 & 0.3015 & & 183 & 13 & 0.3227 & & 549 & 13 & 0.322 \\
\hline & & 54 & 15 & 0.2857 & & 177 & 15 & 0.3121 & & 543 & 15 & 0.319 \\
\hline & & 36 & 16 & 0.1904 & & 123 & 16 & 0.2169 & & 381 & 16 & 0.224 \\
\hline & & 30 & 19 & 0.1587 & & 105 & 19 & 0.1851 & & 327 & 19 & 0.192 \\
\hline & & 24 & 22 & 0.1269 & & 87 & 22 & 0.1534 & & 273 & 22 & 0.160 \\
\hline & & 18 & 31 & 0.0952 & & 81 & 31 & 0.1428 & & 255 & 31 & 0.149 \\
\hline & & 16 & 34 & 0.0846 & & 75 & 34 & 0.1322 & & 237 & 34 & 0.1393 \\
\hline & & 10 & 40 & 0.0529 & & 57 & 40 & 0.1005 & & 183 & 40 & 0.1075 \\
\hline & & 7 & 46 & 0.0370 & & 54 & 46 & 0.0952 & & 177 & 46 & 0.1040 \\
\hline & & 1 & 94 & 0.0053 & & 36 & 49 & 0.0634 & & 123 & 49 & 0.0723 \\
\hline & & & & & & 30 & 58 & 0.0529 & & 105 & 58 & 0.0617 \\
\hline & & & & & & 24 & 67 & 0.0423 & & 87 & 67 & 0.0511 \\
\hline & & & & & & 18 & 94 & 0.0317 & & 81 & 94 & 0.0476 \\
\hline & & & & & & 16 & 103 & 0.0282 & & 75 & 103 & 0.0440 \\
\hline & & & & & & 10 & 121 & 0.0176 & & 57 & 121 & 0.0335 \\
\hline & & & & & & 7 & 139 & 0.0123 & & 54 & 139 & 0.0317 \\
\hline & & & & & & 1 & 283 & 0.0017 & & 36 & 148 & 0.0211 \\
\hline & & & & & & & & & & 30 & 175 & 0.0176 \\
\hline & & & & & & & & & & 10 & 364 & 0.0058 \\
\hline & & & & & & & & & & 1 & 850 & 0.0005 \\
\hline
\end{tabular}


respectively. The code $(49,28)$ is interleaved code of depth 7 , which is formed by interleaving the following 7 codewords from $(7,4)$ code that is (0000000), (1101000), (0000000), (0000000), (0000000), (0000000) and (0000000). on writing them column by column it gives

$$
v^{1}=(01000000100000000000001000000000000000000000000000) \in C_{49} .
$$

In a similar way, codeword of $(343,196)$ and $(2401,1372)$ are obtained by writing column by column 7 codeword of $C_{49}$ and $C_{343}$. Therefore, for decoding a received polynomial in $C_{343}$ one can easily reverse the process and correct errors in the codeword of either $C_{49}$ or $C_{7}$.

\title{
ACKNOWLEDMENT
}

Acknowledgment to FAPESP by financial support 2013/25977-7. The authors would like to thank the anonymous reviewers for their intuitive commentary that signicantly improved the worth of this work.

\begin{abstract}
RESUMO. Neste trabalho, apresentamos um método que estabelece como uma sequência de códigos $\mathrm{BCH}$ não primitivos pode ser obtida através de um dado código $\mathrm{BCH}$ primitivo. Para isso, utilizamos uma técnica de construção diferente da técnica rotineira de códigos BCH e usamos a estrutura de anéis monoidais em vez de anéis de polinômios. Consequentemente, mostramos que existe uma sequência $\left\{C_{b^{j} n}\right\}_{1 \leq j \leq m}$, onde $b^{j} n$ é o comprimento do código $C_{b^{j} n}$, de códigos BCH binários não primitivos em vez de um dado código binário $\mathrm{BCH} C_{n}$ de comprimento $n$. Algoritmos simulados via Mathlab para codificação e decodificação para este tipo de códigos são introduzidos. O algoritmo via o Matlab fornece rotinas para a construção de um código $\mathrm{BCH}$ primitivo, mas impõe várias restrições, como por exemplo, o grau $s$ de um polinômio irredutível primitivo deve ser menor que 16. Este trabalho trata-se de polinômios não-primitivos irredutíveis com grau $b s$, que são maiores do que 16.
\end{abstract}

Palavras-chave: Anel monoidal, códigos $\mathrm{BCH}$, polinômio primitivo, polinômio não-primitivo.

\section{REFERENCES}

[1] A.A. Andrade \& P. Jr. Linear codes over finite rings. TEMA - Trends in Applied and Computational Mathematics, 6(2) (2005), 207-217.

[2] A.A. Andrade, T. Shah \& A. Khan. A note on linear codes over semigroup rings. TEMA - Trends in Applied and Computational Mathematics, 12(2) (2011), 79-89.

[3] A.S. Ansari \& T. Shah. An association between primitive and non-primitive BCH codes using monoid rings. EURASIP - Journal on Wireless Communications and Networking, 38 (2016).

[4] J. Cazaran, A.V. Kelarev, S.J. Quinn \& D. Vertigan. An algorithm for computing the minimum distances of extensions of BCH codes embedded in semigroup rings. Semigroup Forum, 73(3) (2006), 317-329. 
[5] A.V. Kelarev. "Ring constructions and applications". World Scientific Publishing Co. Inc., New York (2002).

[6] A.V. Kelarev \& P. Solé. Error-correcting codes as ideals in group rings. Contemporary Mathematics, 273 (2001), 11-18.

[7] S. Lin \& J. D. J. Costello. "Error control coding: fundamentals and applications". Prentice Hall Professional Technical Reference, New York (1994).

[8] S.R. Nagpaul \& S.K. Jain. "Topics in applied abstract algebra", The Brooks/Cole Series in Advanced Mathematics. New York (2005).

[9] V.S. Pless, W.C. Huffman \& R.A. Brualdi. "Handbook of coding theory". Elsevier, New York (1998).

[10] A. Poli \& L. Huguet. "Error-correcting codes: theory and applications". Prentice-Hall, New York (1992).

[11] T. Shah, Amanullah \& A.A. de Andrade. A decoding procedure which improves code rate and error corrections. Journal of Advanced Research in Applied Mathematics, 4(4) (2012), 37-50.

[12] T. Shah, Amanullah \& A.A. de Andrade. A method for improving the code rate and error correction capability of a cyclic code. Computational and Applied Mathematics, 32(2) (2013), 261-274.

[13] T. Shah \& A.A. de Andrade. Cyclic codes through $B[X], B\left[X ; \frac{1}{k p} Z_{0}\right]$ and $B\left[X ; \frac{1}{p^{k}} Z_{0}\right]$ : a comparison. Journal of Algebra and its Applications, 11(4) (2012), 1250078, 19.

[14] T. Shah \& A.A. de Andrade. Cyclic codes through $B\left[X ; \frac{a}{b} \mathbb{Z}_{0}\right]$, with $\frac{a}{b} \in \mathbb{Q}^{+}$and $b=a+1$, and encoding. Discrete Mathematics, Algorithms and Applications, 4(4) (2012), 1250059, 14.

[15] T. Shah, A. Khan \& A.A. Andrade. Encoding through generalized polynomial codes. Comp. Appl. Math., 30(2) (2011), 349-366.

[16] T. Shah, M. Khan \& A.A. de Andrade. A decoding method of an $n$ length binary BCH code through $(n+1) n$ length binary cyclic code. Anais da Academia Brasileira de Ciências, 85(3) (2013), 863-872.

[17] T. Shah \& A. Shaheen. Cyclic codes as ideals in $F_{2}\left[x ; a N_{0}\right]_{n}, F_{2}[x]_{a n}$, and $F_{2}\left[x ; \frac{1}{b} N_{0}\right]_{a b n}:$ a linkage. U.P.B. Sci. Bull., Series A, 78(3) (2016), 205-220. 